\title{
EXTENSIONS OF FINITE QUANTUM GROUPS BY FINITE GROUPS
}

\author{
NICOLÁS ANDRUSKIEWITSCH AND GASTÓN ANDRÉS GARCÍA
}

\begin{abstract}
Let $G$ be a connected, simply connected complex semisimple Lie group with Lie algebra $\mathfrak{g}$, Cartan matrix $C$ and symmetrized Cartan matrix $C D$. Let $\ell \geq 3$ be an odd integer, relatively prime to $\operatorname{det} C D$. Given an embedding $\sigma$ of a finite group $\Gamma$ on $G$ and a primitive $\ell$-th root of unity $\epsilon$, we construct a central extension $A_{\sigma}$ of the function algebra $\mathbb{C}^{\Gamma}$ by the dual of the Frobenius-Lusztig kernel $\mathbf{u}_{\epsilon}(\mathfrak{g}) ; A_{\sigma}$ is a quotient of the quantized coordinate algebra $\mathcal{O}_{\epsilon}(G)$ and $\operatorname{dim} A_{\sigma}=|\Gamma| \ell^{\operatorname{dim} \mathfrak{g}}$. If $G$ is simple and $\sigma(\Gamma)$ is not central in $G$, then we obtain an infinite family of pairwise non-isomorphic Hopf algebras which are non-semisimple, non-pointed and their duals are also non-pointed. This generalizes the result obtained by E. Müller [M3] for $S L_{2}(\mathbb{C})$.
\end{abstract}

\section{INTRODUCTION}

Let $k$ be an algebraically closed field of characteristic zero. In 1975, I. Kaplansky conjectured that there are finitely many Hopf algebras of a given dimension, up to isomorphism. In 1998, this conjecture was denied by several authors who constructed, by different methods, infinite families of non-isomorphic Hopf algebras of the same dimension. In this direction, the examples given by E. Müller [M3 look quite different from the others. These are non-trivial central extensions of function algebras $\mathbb{C}^{\Gamma}$ of finite subgroups $\Gamma$ of $S L_{2}(\mathbb{C})$ of odd order $\ell$, by the dual of the Frobenius-Lusztig kernel $\mathbf{u}_{\epsilon}\left(\mathfrak{s l}_{2}\right)$, where $\epsilon$ is an $\ell$-th root of unity. These Hopf algebras are nonsemisimple, non-pointed and their duals are non-pointed; they are quotients of the quantized coordinate algebra $\mathcal{O}_{\epsilon}\left(S L_{2}\right)$ and their dimensions are $\ell^{4}$.

In this paper, we generalize the construction given by E. Müller to every connected, simply connected complex simple Lie group $G$. That is, we construct finite-dimensional Hopf algebras $A_{\sigma}$, which are central extensions of the function algebra $\mathbb{C}^{\Gamma}$ of a suitable finite subgroup $\Gamma$ of $G$ by the dual of the Frobenius-Lusztig kernel $\mathbf{u}_{\epsilon}(\mathfrak{g})$ of $\mathfrak{g}$, where $\mathfrak{g}$ is the Lie algebra of $G$. From this follows the title of the present work, since as usual, we consider the category of quantum groups as the category opposite to the category

1991 Mathematics Subject Classification. 16W30, 17 B37.

Research of this paper was begun when G. A. G. was visiting the Mathematisches Institut der Ludwig-Maximilians Universität München under the support of the DAAD. Results in this paper are part of the Ph.D. thesis of G. A. G., written under the advise of N. A. The work was partially supported by CONICET, ANPCyT and Secyt (UNC) . 
of Hopf algebras. We stress that non-abelian extensions of Hopf algebras are difficult to find, except for easy examples like the tensor product. The extensions here are not built in terms of weak actions and cocycles, but arise from the quantum Frobenius morphism.

We begin in Section 2 by studying some general facts about central extensions of Hopf algebras. Specifically, we describe how to construct central extensions of finite-dimensional Hopf algebras from an exact sequence of Hopf algebras $1 \rightarrow B \stackrel{\iota}{\rightarrow} A \stackrel{\pi}{\rightarrow} H \rightarrow 1$, with $B$ central in $A$, and two Hopf algebras epimorphisms $p: B \rightarrow K$ and $r: H \rightarrow L$. We do this in two steps. In the first step we just consider the epimorphism $p: B \rightarrow K$. Let $\mathcal{J}=\operatorname{Ker} p$ and $(\mathcal{J})=A \mathcal{J}$. Then $(\mathcal{J})$ is a Hopf ideal of $A$ and the Hopf algebra $A_{p}=A /(\mathcal{J})$ is given by a pushout and fits into the following commutative diagram, whose rows are exact sequences of Hopf algebras and $B$ and $K$ are central in $A$ and $A_{p}$ respectively.

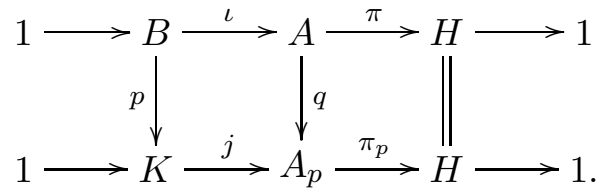

For the second step, we refer to Proposition [2.2.7. In this paper, we perform the first construction in a family of concrete examples, leaving the second construction for future research. Let $G$ be a connected, simply connected complex semisimple Lie group and $\mathfrak{g}$ its Lie algebra. Let $\ell \geq 3$ be an odd integer, prime to 3 if $\mathfrak{g}$ contains a $G_{2}$ component and let $\epsilon$ be a primitive $\ell$-th root of unity. In Section 3 we recall the definition of the quantized coordinate algebra $\mathcal{O}_{\epsilon}(G)$ of $G$ at $\epsilon$. It is well-known that $\mathcal{O}_{\epsilon}(G)$ is a central extension of $\mathcal{O}(G)$, the coordinate algebra of functions on $G$, by the Hopf algebra $H=\mathcal{O}_{\epsilon}(G) / \mathcal{O}(G)^{+} \mathcal{O}_{\epsilon}(G)$; see [DL and BG]. Furthermore, this Hopf algebra $H$ is isomorphic to the dual of the Frobenius-Lusztig kernel $\mathbf{u}_{\epsilon}(\mathfrak{g})$ of $\mathfrak{g}$ at $\epsilon$ and $\mathcal{O}_{\epsilon}(G)$ fits into the exact sequence of Hopf algebras

$$
1 \rightarrow \mathcal{O}(G) \stackrel{\iota}{\rightarrow} \mathcal{O}_{\epsilon}(G) \stackrel{\pi}{\rightarrow} \mathbf{u}_{\epsilon}(\mathfrak{g})^{*} \rightarrow 1
$$

In Section 4, we apply the first general construction to the case in which $A=\mathcal{O}_{\epsilon}(G), B=\mathcal{O}(G)$ and $H=\mathbf{u}_{\epsilon}(\mathfrak{g})^{*}$. Then given a finite group $\Gamma$, in Theorem 4.1.7 we construct for every embedding $\sigma: \Gamma \rightarrow G$, a Hopf algebra $A_{\sigma}$ of dimension $|\Gamma| \ell^{\operatorname{dim} \mathfrak{g}}$. It is a finite-dimensional quotient of $\mathcal{O}_{\epsilon}(G)$ and it is central extension of $\mathbb{C}^{\Gamma}$ by $\mathbf{u}_{\epsilon}(\mathfrak{g})^{*}$, which fits into an exact sequence

$$
1 \rightarrow \mathbb{C}^{\Gamma} \rightarrow A_{\sigma} \rightarrow \mathbf{u}_{\epsilon}(\mathfrak{g})^{*} \rightarrow 1 .
$$

This Hopf algebra $A_{\sigma}$ is non-semisimple and non-pointed. If moreover $\sigma(\Gamma) \nsubseteq \mathbf{T}$, where $\mathbf{T}$ is a maximal torus of $G$ determined by the inclusion of $\mathcal{O}(G)$ in $\mathcal{O}_{\epsilon}(G)$, then its dual is also non-pointed.

Finally we study the isomorphism classes of this type of extensions. Assuming that $G$ is simple and that $\ell$ is relatively prime to the determinant 
of the symmetrized Cartan matrix $C D$, we show in Theorem 4.3.2 that the isomorphisms of the Hopf algebras extensions $A_{\sigma}$ induce an equivalence relation on the set $\operatorname{Emb}(\Gamma, G)$ of embeddings of $\Gamma$ in $G$. This equivalence is given by a triple $(\tau, f, v)$, where $\tau \in \operatorname{Aut}(\Gamma), f$ belongs to a subgroup qAut $(G)$ of $\operatorname{Aut}(G)$ and $v$ is a 1-cocycle of $\Gamma$ with coefficients in a subgroup $\mathbf{T}^{f \sigma}$ of the fixed maximal torus $\mathbf{T}$ of $G$. Let $\sigma \in \operatorname{Emb}(\Gamma, G)$ and assume that $\sigma(\Gamma)$ is not central in $G$. We show in Theorem 4.3.5 that $\sigma$ gives rise to an infinite family of non-semisimple and non-pointed Hopf algebras of dimension $|\Gamma| \ell^{\operatorname{dim} \mathfrak{g}}$, whose duals are also non-pointed. We notice that the proof requires some technical preparations on the cohomology of $\Gamma$ in $\mathbf{T}^{f \sigma}$, and the inequality

$$
\operatorname{dim} G>\operatorname{rk} G+\operatorname{dim} M
$$

for any maximal reductive subgroup $M$ of $G$. As the maximal subalgebras of the simple Lie algebras were classified by Dynkin, we have proved this by inspection in the lists of [D1, D2]. See G2] for details.

We end the paper discussing several explicit examples where the criterium applies.

1.1. Conventions. Our references for the theory of Hopf algebras are $\mathrm{Mo}$ and $[\underline{\mathrm{Sw}}$, for Lie algebras $[\mathrm{Hu}]$ and for quantum groups $[\mathrm{J}]$ and $[\mathrm{BG}$. The antipode of a Hopf algebra $H$ is denoted by $\mathcal{S}$. The Sweedler notation is used for the comultiplication of $H$ but dropping the summation symbol. The set of group-like elements of a coalgebra $C$ is denoted by $G(C)$. We also denote by $C^{+}=\operatorname{Ker} \varepsilon$ the augmentation ideal of $C$, where $\varepsilon: C \rightarrow k$ is the counit

of $C$. Let $A$ and $H$ be Hopf algebras and let $A \stackrel{\pi}{\rightarrow} H$ be a Hopf algebra map. Then $A^{\operatorname{co} \pi}=\{a \in A \mid(\mathrm{id} \otimes \pi) \Delta(a)=a \otimes 1\}$, denotes the subalgebra of right coinvariants and ${ }^{\text {co } \pi} A=\{a \in A \mid(\pi \otimes \mathrm{id}) \Delta(a)=1 \otimes a\}$, denotes the subalgebra of left coinvariants.

A Hopf algebra $H$ is called semisimple, respectively cosemisimple, if it is semisimple as an algebra, respectively if it is cosemisimple as a coalgebra. The sum of all simple subcoalgebras is called the coradical of $H$ and it is denoted by $H_{0}$. If all simple subcoalgebras of $H$ are one-dimensional, then $H$ is called pointed and $H_{0}=k[G(H)]$.

\section{Central extensions of Hopf algebras}

2.1. Preliminaries. In the following we recall some results on quotients and extensions of Hopf algebras, see e. g. [A, AD, Hf, MS, Mo, Sch2].

Definition 2.1.1. AD A sequence of Hopf algebras maps

$$
1 \rightarrow B \stackrel{\iota}{\rightarrow} A \stackrel{\pi}{\rightarrow} H \rightarrow 1,
$$

where 1 denotes the Hopf algebra $k$, is exact if

(i) $\iota$ is injective,

(ii) $\pi$ is surjective,

(iii) $\operatorname{Ker} \pi=A B^{+}$, 
(iv) $B={ }^{\operatorname{co} \pi} A$.

In such a case, $A$ is called an extension of the Hopf algebra $B$ by the Hopf algebra $H$. We usually identify $B$ with its image in $A$ and we simply say that $A$ is a $H$-extension of $B$, if no confusion arises. If the image of $B$ is central in $A$, we say that $A$ is a central extension of $B$.

Let $A$ and $B$ be two Hopf algebras. Denote by $\operatorname{Reg}(B, A)$ the group of linear homomorphisms from $B$ to $A$ which are invertible with respect to the convolution and define

$$
\begin{aligned}
\operatorname{Reg}_{1}(B, A) & =\{\alpha \in \operatorname{Reg}(B, A): \alpha(1)=1\}, \\
\operatorname{Reg}_{\varepsilon}(B, A) & =\{\alpha \in \operatorname{Reg}(B, A): \varepsilon \alpha=\varepsilon\}, \\
\operatorname{Reg}_{1, \varepsilon}(B, A) & =\operatorname{Reg}_{1}(B, A) \cap \operatorname{Reg}_{\varepsilon}(B, A) .
\end{aligned}
$$

Clearly, the sets $\operatorname{Reg}_{1}(B, A), \operatorname{Reg}_{\varepsilon}(B, A)$ and $\operatorname{Reg}_{1, \varepsilon}(B, A)$ are subgroups of $\operatorname{Reg}(B, A)$.

An $A$-comodule algebra is an algebra $C$ that is simultaneously an $A$ comodule whose structural morphism $\rho: C \rightarrow C \otimes A$ is a morphism of algebras. If $R=\{c \in C: \rho(c)=c \otimes 1\}$ is the subalgebra of right coinvariants of $C$, we say that $C$ is an $A$-extension of $R$. An extension of algebras is cleft if there exists $\gamma$ in $\operatorname{Reg}_{1}(A, C)$ such that $\rho \gamma=(\gamma \otimes \mathrm{id}) \Delta$; such $\gamma$ is called a section.

An $A$-module coalgebra is a coalgebra $C$ that is simultaneously an $A$ module whose structural morphism $\mu: A \otimes C \rightarrow C$ is a morphism of coalgebras. If $D=C / A^{+} C$ denotes the coalgebra of coinvariants, we say that $C$ is an $A$-extension of $D$. An extension of coalgebras is cleft if there exists $\xi$ in $\operatorname{Reg}_{\varepsilon}(C, A)$ such that $\xi(a c)=a \xi(c)$ for all $a \in A, c \in C$; such $\xi$ is called a retraction.

The following definition was given by several authors; see for example A, Def. 3.1.14].

Definition 2.1.2. Let $1 \rightarrow B \stackrel{\iota}{\rightarrow} A \stackrel{\pi}{\rightarrow} H \rightarrow 1$ be an exact sequence of Hopf algebras. We say that $A$ is a cleft extension of the Hopf algebra $B$ by the Hopf algebra $H$, if there exist a section $\gamma \in \operatorname{Reg}_{1}(H, A)$ of the algebra extension and a retraction $\xi \in \operatorname{Reg}_{\varepsilon}(A, B)$ of the coalgebra extension satisfying one of the following equivalent conditions for all $a \in A$ :

(i) $\gamma^{-1}(\pi(a))=\mathcal{S}\left(a_{(1)}\right) \xi\left(a_{(2)}\right)$;

(ii) $\gamma(\pi(a))=\xi^{-1}\left(a_{(1)}\right) a_{(2)}$;

(iii) $\xi^{-1}(a)=\gamma\left(\pi\left(a_{(1)}\right)\right) \mathcal{S}\left(a_{(2)}\right)$;

(iv) $\xi(a)=a_{(1)} \gamma^{-1}\left(\pi\left(a_{(2)}\right)\right)$;

(v) $\xi \gamma=\varepsilon_{H} 1_{B}$.

The following result is a consequence of [Sch2, Thm. 2.2].

Theorem 2.1.3. A finite-dimensional Hopf algebra extension is always cleft. 
Recall that a Hopf subalgebra $B$ of a Hopf algebra $A$ is called normal if both $a_{(1)} b \mathcal{S}\left(a_{(2)}\right) \in B$ and $\mathcal{S}\left(a_{(1)}\right) b a_{(2)} \in B$, for all $a \in A, b \in B$. The following proposition was obtained independently in $\mathrm{AD}$ ] and $\mathrm{Sch}$; for a proof see loc. cit. or Mo, Prop. 3.4.3].

Proposition 2.1.4. Let $A$ be a Hopf algebra and let $B$ be a Hopf subalgebra of $A$ such that $A$ is left or right faithfully flat over $B$, and such that $A B^{+}=$ $B^{+} A$. Let $\bar{A}=A / A B^{+}$and consider $A$ as a right and left $\bar{A}$ comodule via $\rho=(\mathrm{id} \otimes \pi) \Delta$ and $\rho^{\prime}=(\pi \otimes \mathrm{id}) \Delta$ respectively, where $\pi: A \rightarrow \bar{A}$ denotes the canonical projection. Then

(a) $B=A^{\text {co } \pi}={ }^{\operatorname{co} \pi} A$.

(b) $B$ is a normal Hopf subalgebra of $A$.

Here is our first basic result.

Proposition 2.1.5. Let $A$ and $K$ be Hopf algebras, $B$ a central Hopf subalgebra of $A$ such that $A$ is left or right faithfully flat over $B$ and $p: B \rightarrow K a$ Hopf algebra epimorphism. Then $H=A / A B^{+}$is a Hopf algebra and $A$ fits into the exact sequence $1 \rightarrow B \stackrel{\iota}{\rightarrow} A \stackrel{\pi}{\rightarrow} H \rightarrow 1$. If we set $\mathcal{J}=\operatorname{Ker} p \subseteq B$, then $(\mathcal{J})=A \mathcal{J}$ is a Hopf ideal of $A$ and $A /(\mathcal{J})$ is the pushout given by the following diagram:

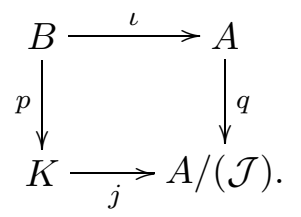

Moreover, $K$ can be identified with a central Hopf subalgebra of $A /(\mathcal{J})$ and $A /(\mathcal{J})$ fits into the exact sequence

$$
1 \rightarrow K \rightarrow A /(\mathcal{J}) \rightarrow H \rightarrow 1
$$

Proof. The first assertion follows directly from Proposition 2.1.4 Since $B$ is central in $A,(\mathcal{J})$ is a two-sided ideal of $A$. Moreover, from the fact that $\varepsilon$ and $\Delta$ are algebra maps and $\mathcal{S}(\mathcal{J}) \subseteq \mathcal{J}$, it follows that $(\mathcal{J})$ is indeed a Hopf ideal. Identify $K$ with $B / \mathcal{J}$. Then the map $j: K \rightarrow A /(\mathcal{J})$ given by $j(b+\mathcal{J})=\iota(b)+(\mathcal{J})$ defines a morphism of Hopf algebras because $\iota$ is a Hopf algebra map. Since $A$ is faithfully flat over $B$, by Sch2, Cor. 1.8] $B$ a direct summand in $A$ as a $B$-module, say $A=B \oplus M$. Then $(\mathcal{J}) \cap B=\mathcal{J} A \cap B=(\mathcal{J} B \oplus \mathcal{J} M) \cap B=(\mathcal{J} \oplus \mathcal{J} M) \cap B=\mathcal{J}$. Thus, if $j(b+\mathcal{J})=0$ then $\iota(b) \in(\mathcal{J})$ and this implies that $b \in(\mathcal{J}) \cap B=\mathcal{J}$ by the equality above. Hence, $j$ is injective.

Let us see now that $A /(\mathcal{J})$ is a pushout: let $C$ be a Hopf algebra and suppose that there exist Hopf algebra maps $\varphi_{1}: K \rightarrow C$ and $\varphi_{2}: A \rightarrow C$ such that $\varphi_{1} p=\varphi_{2} \iota$. We have to show that there exists a unique Hopf algebra map $\phi: A /(\mathcal{J}) \rightarrow C$ such that $\phi q=\varphi_{2}$ and $\phi j=\varphi_{1}$. 


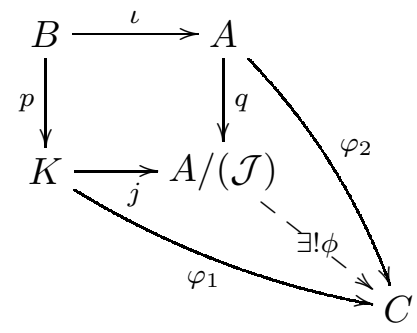

Since $\varphi_{2}((\mathcal{J}))=\varphi_{2}(A \mathcal{J})=\varphi_{2}(A) \varphi_{2}(\iota(\mathcal{J}))=\varphi_{2}(A) \varphi_{1}(p(\mathcal{J}))=0$, there exists a unique Hopf algebra map $\phi: A /(\mathcal{J}) \rightarrow C$ such that $\phi q=\varphi_{2}$. Moreover, let $x \in K$ and $b \in B$ such that $p(b)=x$. Then $\phi j(x)=\phi j p(b)$ $=\phi q \iota(b)=\varphi_{2} \iota(b)=\varphi_{1} p(b)=\varphi_{1}(x)$, from which follows that $\phi j=\varphi_{1}$.

Denote also by $K$ the image of $K$ under $j$. To see that $K$ is central in $A /(\mathcal{J})$ we have to verify that $j(c) \bar{a}=\bar{a} j(c)$ for all $\bar{a} \in A /(\mathcal{J}), c \in K$. Since $p$ is surjective, for all $c \in K$ there exists $b \in B$ such that $p(b)=c$ and since $q$ is an algebra map, it follows that $\bar{a} j(c)=q(a) j(p(b))=q(a) q(\iota(b))=q(a \iota(b))$ $=q(\iota(b) a)=q(\iota(b)) q(a)=j(c) \bar{a}$, because $B$ is central in $A$. In particular, the quotient $\widetilde{H}=[A /(\mathcal{J})] /\left[K^{+}(A /(\mathcal{J}))\right]$ is a Hopf algebra. To see that $A /(\mathcal{J})$ is a central extension of $K$ by $\widetilde{H}$, by Proposition 2.1.4 it is enough to show that $A /(\mathcal{J})$ is flat over $K$ and $K$ is a direct summand of $A /(\mathcal{J})$ as $K$-modules, since by Sch2, Cor. 1.8] this implies that $A /(\mathcal{J})$ is faithfully flat over $K$.

First we show that $A /(\mathcal{J})$ is flat over $K$. Let $M_{1}$ and $M_{2}$ be two right $K$ modules and let $f: M_{1} \rightarrow M_{2}$ be an injective homomorphism. In particular, they admit a $B$-module structure via the map $p: B \rightarrow K$, which we denote by $\bar{M}_{i}$ for $i=1,2$; thus $f$ is an injective homomorphism of $B$-modules. Since $A$ is faithfully flat over $B$, the homomorphism of $A$-modules $f \otimes \mathrm{id}: \bar{M}_{1} \otimes_{B}$ $A \rightarrow \bar{M}_{2} \otimes_{B} A$ is also injective. As $J$ is central in $A$, we have for $i=1,2$ that $\left(\bar{M}_{i} \otimes_{B} A\right)(\mathcal{J})=0$. Then the $A$-modules are also $A /(\mathcal{J})$-modules and $\bar{M}_{i} \otimes_{B} A \simeq M_{i} \otimes_{K} A /(\mathcal{J})$ as $A /(\mathcal{J})$-modules by the construction of $\bar{M}_{i}$. Hence the homomorphism of $A /(\mathcal{J})$-modules $f \otimes \mathrm{id}: M_{1} \otimes_{K} A /(\mathcal{J}) \rightarrow$ $M_{2} \otimes_{K} A /(\mathcal{J})$ is injective and $A /(\mathcal{J})$ is flat over $K$.

As $A=B \oplus M$ as $B$-modules, we have that $(\mathcal{J})=A \mathcal{J}=\mathcal{J} \oplus M \mathcal{J}$, where $M \mathcal{J}$ is a $B$-submodule of $M$ and $\mathcal{J}=B \cap(\mathcal{J} \oplus M \mathcal{J})$. Hence $A /(\mathcal{J})=$ $(B \oplus M) /(\mathcal{J} \oplus M \mathcal{J})=K \oplus(M / M \mathcal{J})$ as $K$-modules, which implies that $K$ is a direct summand of $A /(\mathcal{J})$.

In conclusion, $A /(\mathcal{J})$ fits into an exact sequence of Hopf algebras

$$
1 \rightarrow K \stackrel{j}{\rightarrow} A /(\mathcal{J}) \stackrel{r}{\rightarrow} \widetilde{H} \rightarrow 1 .
$$

Since the map $\Psi: K^{+}(A /(\mathcal{J})) \rightarrow\left(B^{+} A\right) /(\mathcal{J})$ defined by $\Psi(\bar{b} \bar{a})=\overline{b a}$ is a $k$-linear isomorphism, it follows that $\widetilde{H}=(A /(\mathcal{J})) /\left[K^{+}(A /(\mathcal{J}))\right] \simeq$ $(A /(\mathcal{J})) /\left[\left(B^{+} A\right) /(\mathcal{J})\right] \simeq A / B^{+} A=H$ and therefore $A /(\mathcal{J})$ fits into an exact sequence (2.1.5). 
We shall also need the following result.

Proposition 2.1.6. [M3, Prop. 3.4 (c)] Let $1 \rightarrow B \stackrel{\iota}{\rightarrow} A \stackrel{\pi}{\rightarrow} H \rightarrow 1$ be an exact sequence of Hopf algebras. Let $J$ be a Hopf ideal of $A$ of finite codimension and let $\mathcal{J}=B \cap J$. Then the sequence

$$
1 \rightarrow B / \mathcal{J} \rightarrow A / J \rightarrow H / \pi(J) \rightarrow 1
$$

is also exact.

2.2. From quotients to extensions. Let $A, B$ be Hopf algebras such that $B$ is a central Hopf subalgebra of $A$ and $A$ is faithfully flat over $B$. By Proposition 2.1.4 $A$ fits into the exact sequence

$$
1 \rightarrow B \stackrel{\iota}{\rightarrow} A \stackrel{\pi}{\rightarrow} H \rightarrow 1,
$$

where $H=A / B^{+} A$. Let $p: B \rightarrow K$ and $r: H \rightarrow L$ be Hopf algebras epimorphisms. In what follows we construct from the data given by $p$ and $r$, Hopf algebras which are again central extensions.

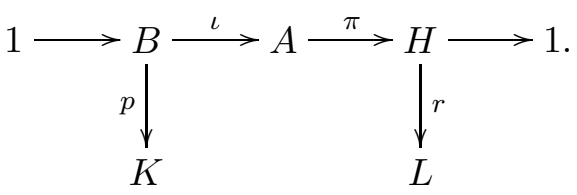

We do this in two steps. In the first step we begin with data associated with the epimorphism $p: B \rightarrow K$. Let $\mathcal{J}=\operatorname{Ker} p$ and $(\mathcal{J})=A \mathcal{J}$. By Proposition 2.1.5, $(\mathcal{J})$ is a Hopf ideal of $A$ and the Hopf algebra $A_{p}=A /(\mathcal{J})$ fits into the following commutative diagram, whose rows are exact sequences of Hopf algebras and $B$ and $K$ are central in $A$ and $A_{p}$ respectively.

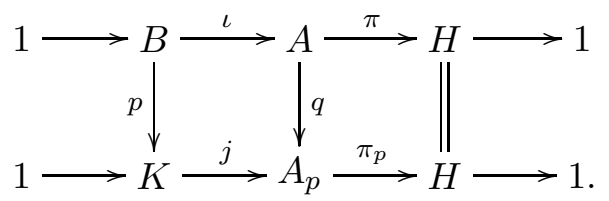

Suppose from now on that $K$ and $H$ are finite-dimensional.

Then $\operatorname{dim} A_{p}$ is also finite, because of the next lemma.

Lemma 2.2.3. Let $K$ and $H$ be finite-dimensional Hopf algebras and suppose that they fit into an exact sequence of Hopf algebras

$$
1 \rightarrow K \rightarrow \mathcal{A} \rightarrow H \rightarrow 1 \text {, }
$$

such that $K$ is central in $\mathcal{A}$. Then $\mathcal{A}$ is also finite-dimensional, the extension is cleft and $\operatorname{dim} \mathcal{A}=\operatorname{dim} K \operatorname{dim} H$.

Proof. Since $K$ is commutative and finite-dimensional, it is semisimple. Thus $\mathcal{A}$ is projective. If $\mathcal{A}$ is infinite-dimensional, by a generalization of [NZ] given in Sch1, Thm. 2.4], $\mathcal{A}$ is a free $K$-module and $A \simeq K^{(I)}$ for some index set $I$. But then $H \simeq \mathcal{A} / K^{+} \mathcal{A} \simeq \mathcal{A} \otimes_{K}\left(K / K^{+}\right) \simeq K^{(I)} \otimes_{K}\left(K / K^{+}\right) \simeq$ $\left(K / K^{+}\right)^{(I)}$ because $\mathcal{A}$ is flat over $K$. Thus card $I$, and a fortiori $\operatorname{dim} \mathcal{A}$ must be finite, a contradiction. The rest follows from Theorem 2.1.3. 
Remark 2.2.4. Let $H$ and $K$ be finite-dimensional Hopf algebras. It seems to be an open question whether a Hopf algebra $\mathcal{A}$ which is an extension of $K$ by $H$ in the sense of Definition [2.1.1] is finite-dimensional.

If $S$ is a subset of $A_{p}$, we denote by $(S)=A_{p} S A_{p}$ the two-sided ideal of $A_{p}$ generated by $S$.

Now we let the epimorphism $r: H \rightarrow L$ enter into the picture. Let $M_{r}=q(\operatorname{Ker} r \pi)=\operatorname{Ker} r \pi_{p}$. Since by Lemma 2.2.3 and Theorem 2.1.3 the $H$-extension $A_{p}$ of $K$ given by the exact sequence

$$
1 \rightarrow K \stackrel{j}{\rightarrow} A_{p} \stackrel{\pi_{p}}{\rightarrow} H \rightarrow 1
$$

is cleft, there exists a retraction $\xi: A_{p} \rightarrow K$ in $\operatorname{Reg}_{\varepsilon}\left(A_{p}, K\right)$ which by Definition 2.1.2 (ii) defines a section $\gamma: H \rightarrow A_{p}$ in $\operatorname{Reg}_{1}\left(H, A_{p}\right)$.

Define $I_{r, \xi}$ to be the minimal Hopf ideal of $A_{p}$ which contains the set

$$
(\mathrm{id}-j \xi)\left(M_{r}\right)=\left\{x-j \xi(x) \mid x \in M_{r}\right\} .
$$

Note that $I_{r, \xi} \subseteq M_{r}$ and $\pi_{p}\left(I_{r, \xi}\right)=\pi_{p}\left(M_{r}\right)$.

Proposition 2.2.7. In the situation above, $\mathcal{I}_{r, \xi}:=I_{r, \xi} \cap K$ is a Hopf ideal of $K$ and $A_{p, r, \xi}:=A_{p} / I_{r, \xi}$ fits into an exact sequence of finite-dimensional Hopf algebras

$$
1 \rightarrow K_{r, \xi} \stackrel{j_{\xi}}{\rightarrow} A_{p, r, \xi} \stackrel{\pi_{\xi}}{\rightarrow} L \rightarrow 1,
$$

where $K_{r, \xi}=K / \mathcal{I}_{r, \xi}$ is central in $A_{p, r, \xi}$.

Proof. By Proposition 2.1.6, the exact sequence (2.2.5) induces an exact sequence of finite-dimensional Hopf algebras

$$
1 \rightarrow K_{r, \xi} \rightarrow A_{p, r, \xi} \rightarrow H / \pi_{p}\left(I_{r, \xi}\right) \rightarrow 1,
$$

where $K_{r, \xi}$ is central in $A_{p, r, \xi}$. Since $M_{r}=\operatorname{Ker}\left(r \pi_{p}\right)$ and $\pi_{p}$ is surjective, it follows that $\pi_{p}\left(M_{r}\right)=\operatorname{Ker} r$. Then $L \simeq H / \operatorname{Ker} r=H / \pi_{p}\left(M_{r}\right)=H / \pi_{p}\left(I_{r, \xi}\right)$ and we get (2.2.8).

After these two steps, we obtain a finite-dimensional Hopf algebra $A_{p, r, \xi}$ associated to the epimorphisms $p: B \rightarrow K$ and $r: H \rightarrow L$, and the retraction $\xi: A_{p} \rightarrow K$, that is, to a triple $(p, r, \xi)$ which makes the following diagram commutative

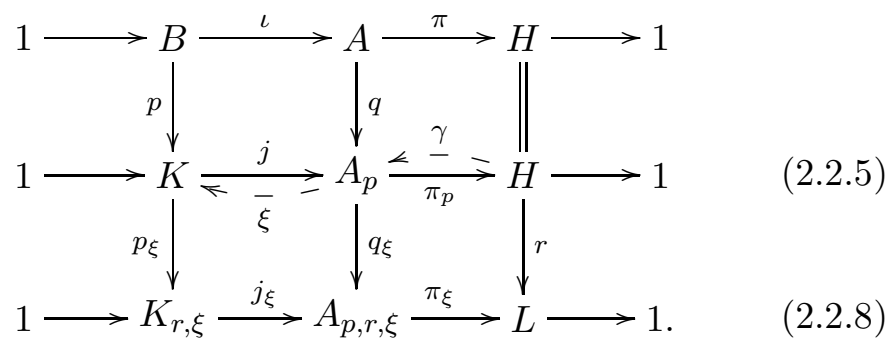


By Proposition 2.1.3, we know that the extension given by the sequence (2.2.8) is cleft, for some section $\bar{\gamma}$ and some retraction $\bar{\xi}$. The next proposition shows how we can choose $\bar{\gamma}$ and $\bar{\xi}$ related to the ones given by the cleft extension $A_{p}$ from (2.2.5).

Proposition 2.2.10. If the extension $A_{p}$ given by the exact sequence (2.2.5) is cleft via $\gamma: H \rightarrow A_{p}$, then the extension $A_{p, r, \xi}$ given by (2.2.8) is cleft via $\bar{\gamma}: L \rightarrow A_{p, r, \xi}$, where $\bar{\gamma}(r(h))=q_{\xi} \gamma(h)$.

Proof. First we have to prove that $\bar{\gamma}$ is well-defined. For this, it is enough to show that $\gamma(\operatorname{Ker} r) \subseteq \operatorname{Ker} q_{\xi}$. Let $t \in \operatorname{Ker} r$ and let $m \in M_{r}=\operatorname{Ker}\left(r \pi_{p}\right)$ such that $\pi_{p}(m)=t$. Since $m \in M_{r}$, we have that $q_{\xi}(m)=p_{\xi} \xi(m)$ and from Definition 2.1.2 (ii), it follows that $m=\xi\left(m_{(1)}\right) \gamma\left(\pi_{p}\left(m_{(2)}\right)\right)$. Then $q_{\xi}\left(\xi\left(m_{(1)}\right)\right) q_{\xi}\left(\gamma\left(\pi_{p}\left(m_{(2)}\right)\right)\right)=q_{\xi}(m)=p_{\xi} \xi(m)$, which implies that $p_{\xi} \xi(m)=$ $\left(p_{\xi} \xi \cdot q_{\xi} \gamma \pi_{p}\right)(m)$. Since $\xi$ is convolution invertible and $p_{\xi}: K \rightarrow K_{r, \xi}$ is a Hopf algebra map, it follows that $p_{\xi} \xi$ is also convolution invertible and therefore $0=\varepsilon(m)=q_{\xi} \gamma \pi_{p}(m)=q_{\xi} \gamma(t)$.

Recall that $A_{p, r, \xi}$ is a right $L$-comodule algebra via $\rho=\left(\mathrm{id} \otimes \pi_{\xi}\right) \Delta$. We show now that $\bar{\gamma}$ is an $L$-comodule map, i. e. $\rho \bar{\gamma}(t)=(\bar{\gamma} \otimes$ id $) \Delta(t)$ for all $t \in L$. Let $t \in L$ and $h \in H$ such that $r(h)=t$. Since $q_{\xi}$ and $r$ are Hopf algebra maps, $\gamma$ is right $H$-colinear and the equality $\pi_{\xi} q_{\xi}=r \pi_{p}$ we obtain

$$
\begin{aligned}
\rho \bar{\gamma}(t) & =\rho \bar{\gamma}(r(h))=\left(\mathrm{id} \otimes \pi_{\xi}\right) \Delta(\bar{\gamma}(r(h))) \\
& =\left(\mathrm{id} \otimes \pi_{\xi}\right) \Delta\left(q_{\xi}(\gamma(h))\right)=\left(\mathrm{id} \otimes \pi_{\xi}\right)\left(q_{\xi} \otimes q_{\xi}\right) \Delta(\gamma(h)) \\
& \left.=\left(q_{\xi} \otimes \pi_{\xi} q_{\xi}\right) \Delta(\gamma(h))\right)=\left(q_{\xi} \otimes r \pi_{p}\right) \Delta(\gamma(h)) \\
& =\left(q_{\xi} \otimes r\right)\left(\mathrm{id} \otimes \pi_{p}\right) \Delta(\gamma(h))=\left(q_{\xi} \otimes r\right)(\gamma \otimes \mathrm{id}) \Delta(h) \\
& =q_{\xi}\left(\gamma\left(h_{(1)}\right)\right) \otimes r\left(h_{(2)}\right)=\bar{\gamma}\left(r\left(h_{(1)}\right)\right) \otimes r\left(h_{(2)}\right) \\
& =\bar{\gamma}\left(r(h)_{(1)}\right) \otimes r(h)_{(2)}=(\bar{\gamma} \otimes \mathrm{id}) \Delta(r(h))=(\bar{\gamma} \otimes \mathrm{id}) \Delta(t) .
\end{aligned}
$$

Finally we prove that $\bar{\gamma} \in \operatorname{Reg}_{1}\left(L, A_{p, r, \xi}\right)$. It is clear that $\bar{\gamma}(1)=\bar{\gamma}(r(1))=$ $q_{\xi} \gamma(1)=q_{\xi}(1)=1$, then we have only to show that $\bar{\gamma}$ is convolution invertible. Let $t \in L$ and $h \in H$ such that $r(h)=t$ and define $\bar{\gamma}^{-1}(t)=q_{\xi} \gamma^{-1}(h)$. As before, one can see that $\bar{\gamma}^{-1}$ is a well-defined function and it is the inverse of $\bar{\gamma}$ with respect to the convolution. Indeed,

$$
\begin{aligned}
\bar{\gamma} * \bar{\gamma}^{-1}(t) & =\bar{\gamma} * \bar{\gamma}^{-1}(r(h))=\bar{\gamma}\left(r(h)_{(1)}\right) \bar{\gamma}^{-1}\left(r(h)_{(2)}\right) \\
& =\bar{\gamma}\left(r\left(h_{(1)}\right)\right) \bar{\gamma}^{-1}\left(r\left(h_{(2)}\right)\right)=q_{\xi} \gamma\left(h_{(1)}\right) q_{\xi} \gamma^{-1}\left(h_{(2)}\right) \\
& =q_{\xi}\left(\gamma\left(h_{(1)}\right) \gamma^{-1}\left(h_{(2)}\right)\right)=q_{\xi}(\varepsilon(h))=\varepsilon(t) .
\end{aligned}
$$

The proof of $\bar{\gamma}^{-1} * \bar{\gamma}=\varepsilon_{L} 1_{A_{p, r, \xi}}$ is similar. If we set for all $a \in A_{p, r, \xi}$, $\bar{\xi}(a)=a_{(1)} \bar{\gamma}^{-1}\left(\pi_{\xi}\left(a_{(2)}\right)\right)$, then by [A, Lemma 3.1.14] we have that $\bar{\xi} \in$ $\operatorname{Reg}_{\varepsilon}\left(A_{p, r, \xi}, K_{r, \xi}\right)$ and satisfies Definition 2.1.2 (iv), which implies that the extension is cleft via $\bar{\gamma}$.

Remark 2.2.11. Since the extension $A_{p}$ given by (2.2.5) is cleft via $\xi$ and $\gamma$, $A_{p} \simeq K^{\tau} \#_{\sigma} H$ as a Hopf algebra, where $\tau: H \rightarrow K \otimes K$ is a two-cocycle 
induced by $\xi$ and $\sigma: H \otimes H \rightarrow K$ is a two-cocycle induced by $\gamma$, see [DT, Thm. 11] and [AD, 3.2.9]. Analogously, the quotient $A_{p, r, \xi}$ is isomorphic to the crossed product $K_{r, \xi}{ }^{\bar{\tau}} \#_{\bar{\sigma}} L$. If we compose these isomorphisms with the quotient $q_{\xi}: A_{p} \rightarrow A_{p, r, \xi}$, we get a Hopf algebra epimorphism $\varphi$ : $K^{\tau} \#_{\sigma} H \rightarrow K_{r, \xi}{ }^{\bar{\tau}} \#{ }_{\bar{\sigma}} L$, given by $\varphi(b \# h)=p_{\xi}(b) \# r(h), b \in K, h \in H$.

Our next goal is to find conditions which help us to describe the ideal $I_{r, \xi}$ and the retraction $\xi$ more explicitly. Let $\left(A_{p}\right)_{0}$ denote the coradical of $A_{p}$. By [Mo Thm. 5.4.2] there exists a coideal $N$ of $A_{p}$ such that $A_{p}=\left(A_{p}\right)_{0} \oplus N$ as $k$-vector spaces. Let $E$ be the subgroup of $G(A)$ given by

$$
E=\{x \in G(A) \mid r \pi(x)=1\}
$$

and let $F=q(E)$. Clearly $G(B) \subseteq E$. Since $B$ is commutative, $K$ is commutative, hence semisimple. Then $K F$ is a semisimple Hopf subalgebra of $A_{p}$, because $\left.\mathcal{S}^{2}\right|_{K F}=\operatorname{id}_{K F}$. Thus $K F \subseteq\left(A_{p}\right)_{0}$, and there exists a coalgebra $C$ such that $\left(A_{p}\right)_{0}=K F \oplus C$. In particular,

$$
A_{p}=K F \oplus D,
$$

where $D=C \oplus N$ is a coideal. Furthermore, we can assume that $\mathcal{S}(D) \subseteq D$, because of the next lemma.

Lemma 2.2.13. Let $\mathcal{A}$ be a Hopf algebra whose antipode $\mathcal{S}$ has finite order, $\mathcal{K}$ a subcoalgebra of $\mathcal{A}$ such that $\mathcal{S}(\mathcal{K})=\mathcal{K}$ and $\pi: \mathcal{A} \rightarrow \mathcal{K}$ a coalgebra projection, i.e. $\pi^{2}=\pi$. Then there exists a coideal $\mathcal{D}$ of $\mathcal{A}$ such that $\mathcal{S}(\mathcal{D})=\mathcal{D}$ and $\mathcal{A}=\mathcal{K} \oplus \mathcal{D}$.

Proof. Let $m=\operatorname{ord} \mathcal{S}$ and $\tilde{\pi}: \mathcal{A} \rightarrow \mathcal{K}$ be the $k$-linear map given by

$$
\tilde{\pi}(a)=\frac{1}{m} \sum_{i=0}^{m} \mathcal{S}^{i} \pi\left(\mathcal{S}^{m-i}(a)\right) \text { for all } a \in \mathcal{A} \text {. }
$$

We claim that $\tilde{\pi}$ is a coalgebra map and $\mathcal{S} \tilde{\pi}=\tilde{\pi} \mathcal{S}$. Clearly, the second assertion follows directly from the definition of $\tilde{\pi}$. If the order $m$ of $\mathcal{S}$ is odd, then $\mathcal{A}$ is commutative and cocommutative. Hence $\mathcal{S}$ and consequently $\tilde{\pi}$ are coalgebra maps. Thus we can assume that $m$ is even. Since the even powers of the antipode are coalgebra maps and $\pi$ is a coalgebra map, it is enough to prove the first assertion for the maps $\mathcal{S}^{i} \pi \mathcal{S}^{m-i}$ where $1 \leq i \leq m$ is odd. In this case, $m-i$ is also odd and the result follows because $\mathcal{S}^{i}$ and $\mathcal{S}^{m-i}$ are anticoalgebra maps. Since by hypothesis $\mathcal{S}(\mathcal{K})=\mathcal{K}$, we have that $\operatorname{Im} \tilde{\pi} \subseteq \operatorname{Im} \pi=\mathcal{K}$. Let $x \in \operatorname{Im} \pi$. Then $\mathcal{S}^{m-i}(x) \in \operatorname{Im} \pi$ for all $1 \leq i \leq m$, and therefore $\pi\left(\mathcal{S}^{m-i}(x)\right)=\mathcal{S}^{m-i}(x)$. This implies that $\tilde{\pi}(x)=x=\pi(x)$ for all $x \in \operatorname{Im} \pi$, and hence $\operatorname{Im} \pi \subseteq \operatorname{Im} \tilde{\pi}$. As usual, $\tilde{\pi}^{2}(x)=\tilde{\pi}(x)$ and $\tilde{\pi}$ is a coalgebra projection. Let $\mathcal{D}=\operatorname{Ker} \tilde{\pi}$. Then $\mathcal{D}$ is a coideal of $\mathcal{A}$ which satisfies that $\mathcal{A}=\mathcal{K} \oplus \mathcal{D}$ and $\mathcal{S}(\mathcal{D})=\mathcal{D}$ since $\tilde{\pi}$ commutes $\mathcal{S}$.

We show now that in some special cases the two-sided ideal $\left((\mathrm{id}-j \xi)\left(M_{r}\right)\right)$ of $A_{p}$ is a Hopf ideal. 
Proposition 2.2.14. Let $D$ be as in (2.2.12). Suppose that $\xi(a)=0$, for all $a \in D \cap M_{r}$ and the map $\left.\xi\right|_{F}: F \rightarrow G(K)$ defines a group homomorphism. Then $I_{r, \xi}=\left((\mathrm{id}-j \xi)\left(M_{r}\right)\right)$.

Proof. Since $\mathcal{S}(D)=D$, we have that $\mathcal{S}\left(D \cap M_{r}\right) \subseteq D \cap M_{r}$, because $M_{r}$ is already a Hopf ideal. Therefore, the two-sided ideal $I_{r}:=\left(D \cap M_{r}\right)$ is a Hopf ideal and coincides with $\left((\mathrm{id}-j \xi)\left(D \cap M_{r}\right)\right)$. On the other hand, if $K F^{+}$denotes the Hopf ideal of $K F$ given by $K\left(k[F]^{+}\right)$, then $K F \cap M_{r}=$ $(K F)^{+}=K^{+} F+K F^{+}$and

$$
\left((\mathrm{id}-j \xi)\left(K F \cap M_{r}\right)\right)=\left((\mathrm{id}-j \xi)\left(K F^{+}\right)\right)=((\mathrm{id}-j \xi)(F)),
$$

since $\xi$ is a $K$-module map such that $\xi_{K}=\mathrm{id}_{K}$. Thus, if $\left.\xi\right|_{F}$ defines a group homomorphism it follows that $I_{\xi}:=((\mathrm{id}-j \xi)(F))$ is a Hopf ideal and $I_{r, \xi}=I_{r}+I_{\xi}=\left((\mathrm{id}-j \xi)\left(M_{r}\right)\right)$.

The next proposition shows that under certain conditions, there exist retractions $\xi$ which satisfy the hypothesis of Proposition 2.2.14.

Proposition 2.2.15. Assume that $A_{p}=K F \oplus D$, with $D$ a $K$-module coideal. Let $\beta: F \rightarrow G(K)$ be a group homomorphism such that $\left.\beta q\right|_{\iota(G(B))}=$ $\left.p\right|_{G(B)}$. Then there is a retraction $\xi: A_{p} \rightarrow K$ such that $\left.\xi\right|_{D}=0$ and $\left.\xi\right|_{F}=\beta$.

Proof. Since $A_{p}=K F \oplus D$ as $K$-modules, it is enough to define $\xi$ on $D$ and $K F$. Since $\operatorname{dim} K F<\infty, K F$ is a free $K$-module of rank $m=|F| /|G(K)|$ by Nichols-Zoeller. Let $\left\{e_{1}, \ldots, e_{m}\right\}$ be a basis of $K F$ which consists of the a set of representatives of the left cosets $F / G(K)$ such that $e_{1}=1$. Define $\widehat{\beta}$ to be the unique $K$-map given by $\widehat{\beta}\left(e_{i}\right)=\beta\left(e_{i}\right)$. Then set $\left.\xi\right|_{D}=0$ and define $\left.\xi\right|_{K F}=\hat{\beta}$. Clearly, $\xi$ is a $K$-module map such that $\xi(1)=\hat{\beta}(1)=\beta(1)=1$ and $\xi(a)=\xi(a \cdot 1)=\hat{\beta}(a \cdot 1)=a \hat{\beta}(1)=a$ for all $a \in K$.

Hence, to prove that $\xi$ is a section we have to show that $\xi$ is a convolution invertible map. Since $A_{p}$ admits a decomposition $A_{p}=K F \oplus D$, where $D$ is a coideal and a $K$-module, it suffices to define the inverse for $\xi$ on $K F$. Thus define $\left.\xi^{-1}\right|_{K F}=\hat{\beta}^{-1}$, where $\hat{\beta}^{-1}$ is the $k$-linear map given by $\hat{\beta}^{-1}(a e)=\mathcal{S}(a) \beta^{-1}(e)$ for all $a \in K$ and $e \in\left\{e_{i}\right\}_{1 \leq i \leq m}$. Then for all $a \in K$ and $e \in\left\{e_{i}\right\}_{1 \leq i \leq m}$ we have

$$
\hat{\beta} * \hat{\beta}^{-1}(a e)=\hat{\beta}\left(a_{(1)} e\right) \hat{\beta}^{-1}\left(a_{(2)} e\right)=a_{(1)} \beta(e) \mathcal{S}\left(a_{(2)}\right) \beta^{-1}(e)=\varepsilon(a e) .
$$

Similarly $\hat{\beta}^{-1} * \hat{\beta}=\varepsilon 1$; thus $\hat{\beta}$ is a convolution invertible $K$-module map.

2.3. On the isomorphisms of the obtained extensions. In this section we study some properties of the extensions constructed in Subsection 2.2 which will be needed later in Section 4. We define first the Hopf center of a Hopf algebra, which always exists by [A, Cor. 2.2.2].

Definition 2.3.1. [A] Def. 2.2.3] The Hopf center of a Hopf algebra $A$ is the maximal central Hopf subalgebra $\mathcal{Z}(A)$ of $A$. 
Proposition 2.3.2. For $i=1,2$ let $1 \rightarrow K_{i} \rightarrow A_{i} \rightarrow L_{i} \rightarrow 1$ be an exact sequence of Hopf algebras such that $K_{i}=\mathcal{Z}\left(A_{i}\right)$. Suppose that $\omega: A_{1} \rightarrow A_{2}$ is a Hopf algebra isomorphism. Then there exist isomorphisms $\underline{\omega}: K_{1} \rightarrow K_{2}$ and $\bar{\omega}: L_{1} \rightarrow L_{2}$ such that the following diagram commutes

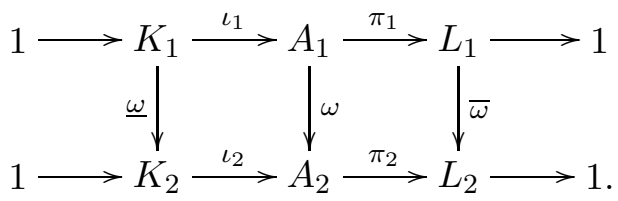

Proof. As $K_{1}=\mathcal{Z}\left(A_{1}\right)$ and $\omega$ is surjective, $\omega\left(K_{1}\right)$ is a central Hopf subalgebra of $A_{2}$. Then $\omega\left(K_{1}\right) \subseteq \mathcal{Z}\left(A_{2}\right)=K_{2}$. Analogously $\omega^{-1}\left(K_{2}\right) \subseteq K_{1}$ and therefore $\omega\left(K_{1}\right)=K_{2}$. Hence, the Hopf algebra map $\underline{\omega}: K_{1} \rightarrow K_{2}$ given by $\underline{\omega}=\left.\omega\right|_{K_{1}}$ is an isomorphism. Since $L_{i}=A / K_{i}^{+} A_{i}$ for $1 \leq i \leq 2$ and $\omega\left(K_{1}\right)=$ $K_{2}, \omega$ induces an isomorphism of Hopf algebras $\bar{\omega}: L_{1} \rightarrow L_{2}$ given by the formula $\bar{\omega}\left(\pi_{1}(a)\right)=\pi_{2}(\omega(a))$ for all $a \in A$. Indeed, if $\bar{\omega}\left(\pi_{1}(a)\right)=0$, then $\omega(a) \in K_{2}^{+} A_{2}=\operatorname{Ker} \pi_{2}$. But since $K_{2}^{+} A_{2}=\omega\left(K_{1}\right)^{+} \omega\left(A_{1}\right)=\omega\left(K_{1}^{+} A_{1}\right)$, there exists $b \in K_{1}^{+} A_{1}$ such that $\omega(a)=\omega(b)$. Since $\omega$ is injective, $a=b \in$ $K_{1}^{+} A_{1}=\operatorname{Ker} \pi_{1}$ and $\underline{\omega}$ is injective. The surjectivity of $\bar{\omega}$ follows from that of $\omega$. Finally, the diagram is commutative by definition of $\underline{\omega}$ and $\bar{\omega}$.

Here is a condition that implies the hypothesis of Proposition 2.3.2.

Lemma 2.3.3. [A 3.3.9] Let $1 \rightarrow K \stackrel{\iota}{\rightarrow} A \stackrel{\pi}{\rightarrow} L \rightarrow 1$ be an exact sequence of finite-dimensional Hopf algebras, with $K$ central in $A$. If $\mathcal{Z}(L)=k$, then $\mathcal{Z}(A)=K$.

Proof. Since $K$ is central in $A$, we have that $K \subseteq \mathcal{Z}(A)$. As $\pi$ is surjective, $\pi(\mathcal{Z}(A))$ is central in $L$ and therefore contained in $\mathcal{Z}(L)=k$. Hence $\left.\pi\right|_{\mathcal{Z}(A)}=$ $\left.\varepsilon\right|_{\mathcal{Z}(A)}$, which implies that $\mathcal{Z}(A) \subseteq{ }^{\operatorname{co} \pi} A=K$.

We give now a sufficient condition for two Hopf algebras constructed via the pushout to be isomorphic. Consider the exact sequence (2.2.1) and let $p_{1}: B \rightarrow K_{1}$ and $p_{2}: B \rightarrow K_{2}$ be two Hopf algebras epimorphisms. Then by Proposition 2.1.5 we can build two Hopf algebras $A_{1}:=A_{p_{1}}$ and $A_{2}:=A_{p_{2}}$, such that $K_{i}$ is central in $A_{i}$ and they fit into a commutative diagram:

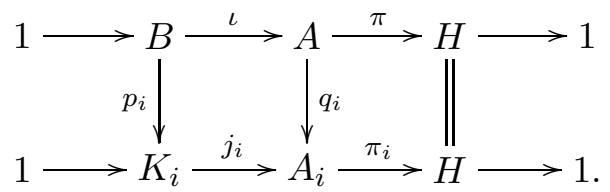

Lemma 2.3.5. Let $f: K_{1} \rightarrow K_{2}$ be a Hopf algebra isomorphism such that $f p_{1}=p_{2}$. Then the Hopf algebras $A_{1}$ and $A_{2}$ are isomorphic. 
Proof. Since $f p_{1}=p_{2}, q_{2} \iota=j_{2} p_{2}=j_{2} f p_{1}$. Since $A_{1}$ is a pushout, there is a unique Hopf algebra map $\omega: A_{1} \rightarrow A_{2}$ such that $\omega q_{1}=q_{2}$ and $\omega j_{1}=j_{2} f$.

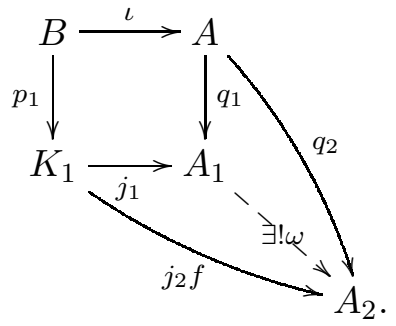

Similarly, there is a unique Hopf algebra map $\omega^{-1}: A_{2} \rightarrow A_{1}$ with analogous properties, which must be the inverse of $\omega$.

We end this section with the following theorem which gives under certain hypothesis a characterization of the isomorphism classes of these type of extensions. First, we need some definitions: We say that the central $H$ extension $A$ of $B$ satisfies

$(L)$ if every automorphism $f$ of $H$ can be lifted to an automorphism $F$ of $A$ such that $\pi F=f \pi$, and

$(Z)$ if $\mathcal{Z}(H)=k$.

Let $f \in \operatorname{Aut}(H)$. If $(L)$ and $(Z)$ are satisfied, then by Lemma 2.3.3. $B=\mathcal{Z}(A)$ and $g=\left.F\right|_{B}$ is an automorphism of $B$. We denote by qut $(B)$ the subgroup of the group $\operatorname{Aut}(B)$ of Hopf algebra automorphisms generated by these automorphisms.

Theorem 2.3.6. Suppose that $(L)$ and $(Z)$ hold. If two Hopf algebras $A_{1}$ and $A_{2}$ as in (2.3.4) are isomorphic then there is a triple $(\underline{\omega}, g, u)$ with

(a) $\underline{\omega}: K_{1} \rightarrow K_{2}$ is an isomorphism,

(b) $g \in \mathrm{qAut}(B)$,

(c) $u \in \operatorname{Reg}_{1, \varepsilon}\left(A, K_{2}\right)$ is an algebra map such that

$$
\begin{aligned}
\underline{\omega}\left(p_{1}(b)\right) & =p_{2}\left(g\left(b_{(1)}\right)\right) u\left(\iota\left(b_{(2)}\right)\right), \\
\Delta(u(a)) & =u\left(a_{(2)}\right) \otimes q_{2}\left(F\left(\mathcal{S}\left(a_{(1)}\right) a_{(3)}\right)\right) u\left(a_{(4)}\right),
\end{aligned}
$$

for all $b \in B$ and $a \in A$, where $F \in \operatorname{Aut}(A)$ is induced by $\bar{\omega}$ with $F \iota=\iota g$.

Conversely, if $\operatorname{dim} K_{1}$ and $\operatorname{dim} H$ are finite and there exists such a triple, then $A_{1}$ and $A_{2}$ are isomorphic.

Proof. Let $\omega: A_{1} \rightarrow A_{2}$ be an isomorphism of Hopf algebras. Since by assumption $\mathcal{Z}(H)=k$, from Lemma 2.3.3 it follows that $\mathcal{Z}\left(A_{i}\right)=K_{i}$ for $1 \leq$ $i \leq 2$. Thus by Proposition 2.3.2 $\omega$ induces an isomorphism $\underline{\omega}: K_{1} \rightarrow K_{2}$ and an automorphism $\bar{\omega} \in \operatorname{Aut}(H)$. Then there exists an automorphism $F$ of $A$ such that $\pi F=\bar{\omega} \pi$ and the map given by $g=\left.F\right|_{B}$ is an automorphism of $B$ such that $F \iota=\iota g$. Define $u: A \rightarrow A_{2}$ to be the $k$-linear map given by

$$
u(a)=q_{2}\left(F\left(\mathcal{S}\left(a_{(1)}\right)\right)\right) \omega\left(q_{1}\left(a_{(2)}\right)\right) \text { for all } a \in A,
$$


that is, $u=q_{2} F \mathcal{S} * \omega q_{1}$, the convolution product between the maps $q_{2} F \mathcal{S}$ and $\omega q_{1}$. Since these maps are convolution invertible with inverses $q_{2} F$ and $\omega q_{1} \mathcal{S}$ respectively, $u$ is also convolution invertible with inverse $\omega q_{1} \mathcal{S} * q_{2} F$.

We claim that $u \in \operatorname{Reg}_{1, \varepsilon}\left(A, K_{2}\right)$, it is an algebra map and satisfies (2.3.7) and (2.3.8). Indeed, it is clear that $u(1)=1$ and $\varepsilon(u(a))=\varepsilon(a)$ for all $a \in A$. To prove that $\operatorname{Im} u \subseteq K_{2}={ }^{\operatorname{co} \pi_{2}} A_{2}$, let $a \in A$; then

$$
\begin{aligned}
& \left(\pi_{2} \otimes \mathrm{id}\right) \Delta(u(a))=\left(\pi_{2} \otimes \mathrm{id}\right) \Delta\left(q_{2}\left(F\left(\mathcal{S}\left(a_{(1)}\right)\right)\right) \omega\left(q_{1}\left(a_{(2)}\right)\right)\right) \\
& =\pi_{2}\left(q_{2}\left(F\left(\mathcal{S}\left(a_{(2)}\right)\right)\right) \omega\left(q_{1}\left(a_{(3)}\right)\right)\right) \otimes q_{2}\left(F\left(\mathcal{S}\left(a_{(1)}\right)\right)\right) \omega\left(q_{1}\left(\left(a_{(4)}\right)\right)\right) \\
& =\pi_{2}\left(q_{2}\left(F\left(\mathcal{S}\left(a_{(2)}\right)\right)\right) \pi_{2}\left(\omega\left(q_{1}\left(a_{(3)}\right)\right)\right) \otimes q_{2}\left(F\left(\mathcal{S}\left(a_{(1)}\right)\right)\right) \omega\left(q_{1}\left(\left(a_{(4)}\right)\right)\right)\right) \\
& \left.=\pi\left(F\left(\mathcal{S}\left(a_{(2)}\right)\right)\right) \bar{\omega} \pi_{1}\left(q_{1}\left(a_{(3)}\right)\right) \otimes q_{2}\left(F\left(\mathcal{S}\left(a_{(1)}\right)\right)\right) \omega\left(q_{1}\left(\left(a_{(4)}\right)\right)\right)\right) \\
& \left.=\bar{\omega} \pi\left(\mathcal{S}\left(a_{(2)}\right)\right) \bar{\omega} \pi\left(a_{(3)}\right) \otimes q_{2}\left(F\left(\mathcal{S}\left(a_{(1)}\right)\right)\right) \omega\left(q_{1}\left(\left(a_{(4)}\right)\right)\right)\right) \\
& \left.=\varepsilon\left(a_{(2)}\right) \otimes q_{2}\left(F\left(\mathcal{S}\left(a_{(1)}\right)\right)\right) \omega\left(q_{1}\left(\left(a_{(3)}\right)\right)\right)\right) \\
& \left.=1 \otimes q_{2}\left(F\left(\mathcal{S}\left(a_{(1)}\right)\right)\right) \omega\left(q_{1}\left(\left(a_{(2)}\right)\right)\right)\right)=1 \otimes u(a) .
\end{aligned}
$$

We prove now $u$ is an algebra map. Let $a, b \in A$, then

$$
\begin{aligned}
u(a b) & =q_{2}\left(F\left(\mathcal{S}\left((a b)_{(1)}\right)\right)\right) \omega\left(q_{1}\left((a b)_{(2)}\right)\right) \\
& =q_{2}\left(F\left(\mathcal{S}\left(a_{(1)} b_{(1)}\right)\right)\right) \omega\left(q_{1}\left(a_{(2)} b_{(2)}\right)\right) \\
& =q_{2}\left(F\left(\mathcal{S}\left(b_{(1)}\right) \mathcal{S}\left(a_{(1)}\right)\right)\right) \omega\left(q_{1}\left(a_{(2)}\right)\right) \omega\left(q_{1}\left(b_{(2)}\right)\right) \\
& =q_{2}\left(F\left(\mathcal{S}\left(b_{(1)}\right)\right)\right) q_{2}\left(F\left(\mathcal{S}\left(a_{(1)}\right)\right)\right) \omega\left(q_{1}\left(a_{(2)}\right)\right) \omega\left(q_{1}\left(b_{(2)}\right)\right) \\
& =q_{2}\left(F\left(\mathcal{S}\left(b_{(1)}\right)\right)\right) u(a) \omega\left(q_{1}\left(b_{(2)}\right)\right) \\
& =u(a)\left(q_{2}\left(F\left(\mathcal{S}\left(b_{(1)}\right)\right)\right) \omega\left(q_{1}\left(b_{(2)}\right)\right)\right)=u(a) u(b),
\end{aligned}
$$

since $u(a) \in K_{2}$ and $K_{2}$ is central in $A_{2}$. This implies that $u$ is an algebra map. Finally, let us prove that $u$ satisfies equations (2.3.7) and (2.3.8): Let $b \in B$, then $u(\iota(b))=q_{2}\left(F\left(\mathcal{S}\left(\iota\left(b_{(1)}\right)\right)\right)\right) \omega\left(q_{1}\left(\iota\left(b_{(2)}\right)\right)\right)$ and therefore

$$
\underline{\omega}\left(p_{1}(b)\right)=\omega\left(q_{1}(\iota(b))\right)=q_{2}\left(F\left(\iota\left(b_{(1)}\right)\right)\right) u\left(\iota\left(b_{(2)}\right)\right)=p_{2}\left(g\left(b_{(1)}\right)\right) u\left(\iota\left(b_{(2)}\right)\right) .
$$

For the second equation, let $a \in A$, then

$$
\begin{aligned}
\Delta(u(a)) & =\left(q_{2}\left(F\left(\mathcal{S}\left(a_{(1)}\right)\right)\right) \omega\left(q_{1}\left(a_{(2)}\right)\right)\right)_{(1)} \otimes\left(q_{2}\left(F\left(\mathcal{S}\left(a_{(1)}\right)\right)\right) \omega\left(q_{1}\left(a_{(2)}\right)\right)\right)_{(2)} \\
& =q_{2}\left(F\left(\mathcal{S}\left(a_{(2)}\right)\right)\right) \omega\left(q_{1}\left(a_{(3)}\right)\right) \otimes q_{2}\left(F\left(\mathcal{S}\left(a_{(1)}\right)\right)\right) \omega\left(q_{1}\left(\left(a_{(4)}\right)\right)\right) \\
& =u\left(a_{(2)}\right) \otimes q_{2}\left(F\left(\mathcal{S}\left(a_{(1)}\right)\right) \omega\left(q_{1}\left(a_{(3)}\right)\right)\right. \\
& =u\left(a_{(2)}\right) \otimes q_{2}\left(F\left(\mathcal{S}\left(a_{(1)}\right)\right)\right) q_{2}\left(F\left(a_{(3)}\right)\right) u\left(a_{(4)}\right) \\
& =u\left(a_{(2)}\right) \otimes q_{2}\left(F\left(\mathcal{S}\left(a_{(1)}\right) a_{(3)}\right)\right) u\left(a_{(4)}\right)
\end{aligned}
$$


Let us prove the converse. Let $(\underline{\omega}, g, u)$ be a triple that satisfies $(a),(b)$ and $(c)$ and let $F \in \operatorname{Aut}(A), \bar{\omega} \in \operatorname{Aut}(H)$ such that $\left.F\right|_{B}=g$ and $F \pi=\bar{\omega} \pi$. Define $\varphi: A \rightarrow A_{2}$ to be the $k$-linear map given by

$$
\varphi(a)=q_{2}\left(F\left(a_{(1)}\right)\right) u\left(a_{(2)}\right) \text { for all } a \in A .
$$

As $K_{2}$ is central in $A_{2}$ and $u \in \operatorname{Reg}_{1, \varepsilon}\left(A, K_{2}\right)$ is an algebra map which satisfies equation (2.3.8), it follows that $\varphi$ is a Hopf algebra map. Moreover, by equation (2.3.7) we have that

$$
\varphi(\iota(b))=j_{2}\left(p_{2}\left(g\left(b_{(1)}\right)\right) u\left(\iota\left(b_{(2)}\right)\right)\right)=j_{2}\left(\underline{\omega}\left(p_{1}(b)\right)\right) \text { for all } b \in B .
$$

Since $A_{1}$ is given by a pushout, there exists a unique Hopf algebra map $\omega: A_{1} \rightarrow A_{2}$ such that the following diagram commutes:

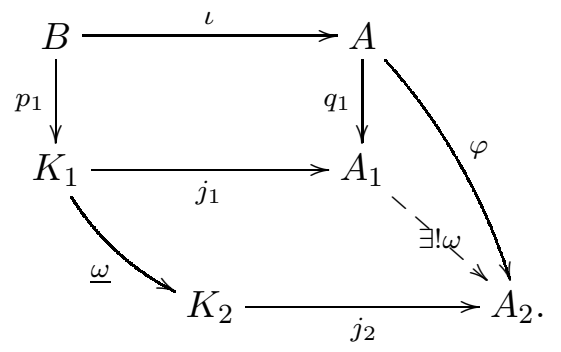

In particular, $j_{2} \underline{\omega}=\omega j_{1}$ and $\bar{\omega} \pi_{1}=\pi_{2} \omega$, since for all $a \in A$ :

$$
\begin{aligned}
\pi_{2} \omega\left(q_{1}(a)\right) & =\pi_{2} \varphi(a)=\pi_{2}\left(q_{2}\left(F\left(a_{(1)}\right)\right) u\left(a_{(2)}\right)\right)=\pi_{2}\left(q_{2}\left(F\left(a_{(1)}\right)\right)\right) \pi_{2}\left(u\left(a_{(2)}\right)\right) \\
& =\pi\left(F\left(a_{(1)}\right)\right) \varepsilon\left(a_{(2)}\right)=\bar{\omega}(\pi(a))=\bar{\omega}\left(\pi_{1}\left(q_{1}(a)\right)\right) .
\end{aligned}
$$

Thus, both exact sequences fit into a commutative diagram

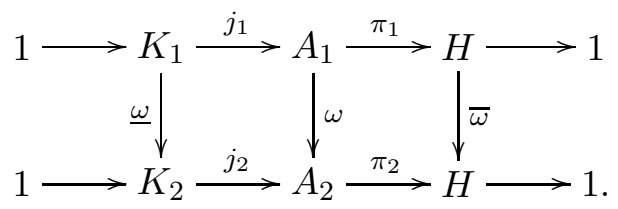

If $\operatorname{dim} K_{1}$ and $\operatorname{dim} H$ are finite, then $\operatorname{dim} A_{1}=\operatorname{dim} A_{2}$ are also finite by Proposition 2.2.3. Since the diagram (2.3.9) is commutative, it follows that

$$
\begin{aligned}
\operatorname{dim} \omega\left(A_{1}\right) & =\operatorname{dim} \underline{\omega}\left(K_{1}\right) \operatorname{dim}\left(\omega\left(A_{1}\right) / \omega\left(K_{1}\right)^{+} \omega\left(A_{1}\right)\right)=\operatorname{dim} \underline{\omega}\left(K_{1}\right) \operatorname{dim} \bar{\omega}(H) \\
& =\operatorname{dim} K_{2} \operatorname{dim} H=\operatorname{dim} A_{2},
\end{aligned}
$$

which implies that $\omega$ is an isomorphism.

\section{Quantized Coordinate Algebras}

Let $G$ be a connected, simply connected complex semisimple Lie group with Lie algebra $\mathfrak{g}$. In this section we recall the definition of the quantized coordinate algebra of $G$ and that it is a central extension of $\mathcal{O}(G)$, the coordinate algebra of functions on $G$. Let $R=\mathbb{Q}\left[q, q^{-1}\right], q$ an indeterminate. In [DL, De Concini and Lyubashenko construct an integral form $\Gamma(\mathfrak{g})$ of the quantized enveloping algebra $U_{q}(\mathfrak{g})$, which is a Hopf $R$-algebra. Using the 
representation theory of $\Gamma(\mathfrak{g})$, they define an $R$-subalgebra of the Hopf dual of $\Gamma(\mathfrak{g})$, obtaining a Hopf $R$-algebra $R_{q}[G]$.

3.1. Definitions. Let $\ell \geq 3$ be an odd integer, prime to 3 if $\mathfrak{g}$ contains a $G_{2}$-component, and let $\epsilon$ be a complex primitive $\ell$-th root of 1 . If $p_{\ell}(q) \in R$ denotes the $\ell$-th cyclotomic polynomial, then $R /\left[p_{\ell}(q) R\right] \simeq \mathbb{Q}(\epsilon)$. We start with the data associated with $\mathfrak{g}$. Let $n$ be the rank of $\mathfrak{g}$ and $C=\left(a_{i j}\right)$ be the Cartan matrix of $\mathfrak{g}$ corresponding to some choice of a Cartan subalgebra $\mathfrak{h}$ and simple roots $\Delta=\left\{\alpha_{1}, \ldots, \alpha_{n}\right\}$. Let $\Phi$ denote the root system and $W$ the Weyl group. The root lattice of $\mathfrak{g}$ is defined by $Q=\mathbb{Z} \Phi=\bigoplus_{i=1}^{n} \mathbb{Z} \alpha_{i} \subseteq \mathfrak{h}^{*}$. The fundamental weights $\varpi_{1}, \ldots, \varpi_{n} \in \mathfrak{h}^{*}$ are defined by the conditions $\left(\varpi_{i}, \alpha_{j}\right)=d_{i} \delta_{i j}$ for all $1 \leq i, j \leq n$, where $(-,-)$ is the positive definite symmetric bilinear form on $\mathfrak{h}^{*}$ induced by the Killing form of $\mathfrak{g}$ and $d_{i}=$ $\frac{\left(\alpha_{i}, \alpha_{i}\right)}{2} \in\{1,2,3\}$. The weight lattice is $P=\bigoplus_{i=1}^{n} \mathbb{Z} \varpi_{i} \subseteq \mathfrak{h}^{*}$. It can be seen that $\alpha_{j}=\sum_{i=1}^{n} a_{i j} \varpi_{i}$ for $1 \leq j \leq n$, so that $Q \subseteq P$.

Definition 3.1.1. Let $L$ be a lattice with $Q \subseteq L \subseteq P$. The quantized enveloping algebra $U_{q}(\mathfrak{g}, L)$ of $\mathfrak{g}$ is the $\mathbb{Q}(q)$-algebra with generators $E_{1}, \ldots, E_{n}$, $F_{1}, \ldots, F_{n}$ and $\left\{K_{\lambda} \mid \lambda \in M\right\}$, satisfying the following relations for $\lambda, \mu \in M$ and $1 \leq i, j \leq n$ :

$$
\begin{gathered}
K_{0}=1 \quad K_{\lambda} K_{\mu}=K_{\lambda+\mu} \\
K_{\lambda} E_{j} K_{-\lambda}=q^{\left(\lambda, \alpha_{j}\right)} E_{j}, \quad K_{\lambda} F_{j} K_{-\lambda}=q^{-\left(\lambda, \alpha_{j}\right)} F_{j}, \\
E_{i} F_{j}-F_{j} E_{i}=\delta_{i j} \frac{K_{\alpha_{i}}-K_{\alpha_{i}}^{-1}}{q_{i}-q_{i}^{-1}} \\
\sum_{l=0}^{1-a_{i j}}(-1)^{l}\left[\begin{array}{c}
1-a_{i j} \\
l
\end{array}\right]_{q_{i}} E_{i}^{1-a_{i j}-l} E_{j} E_{i}^{l}=0 \quad(i \neq j), \\
\sum_{l=0}^{1-a_{i j}}(-1)^{l}\left[\begin{array}{c}
1-a_{i j} \\
l
\end{array}\right]_{q_{i}} F_{i}^{1-a_{i j}-l} F_{j} F_{i}^{l}=0 \quad(i \neq j) .
\end{gathered}
$$

When $M=Q$, the algebra $U_{q}(\mathfrak{g}, L)=U_{q}(\mathfrak{g}, Q)$ is called the adjoint form of the quantized enveloping algebra and it is usually denote by $U_{q}(\mathfrak{g})$. At the other extreme, when $M=P$, the resulting quantized enveloping algebra is called the simply connected form and is denoted $\check{U}_{q}(\mathfrak{g})$.

For $t, m \in \mathbb{N}_{0}$ and $u \in \mathbb{Q}(q) \backslash\{0, \pm 1\}$ we denote:

$$
\begin{aligned}
{[t]_{u}: } & =\frac{u^{t}-u^{-t}}{u-u^{-1}}, \quad[t]_{u} !:=[t]_{u}[t-1]_{u} \cdots[1]_{u}, \quad\left[\begin{array}{c}
m \\
t
\end{array}\right]_{u}:=\frac{[m]_{u} !}{[t]_{u} ![m-t]_{u} !} \\
(t)_{u} & :=\frac{u^{t}-1}{u-1}, \quad(t)_{u} !:=(t)_{u}(t-1)_{u} \cdots(1)_{u}, \quad\left(\begin{array}{c}
m \\
t
\end{array}\right)_{u}:=\frac{(m)_{u} !}{(t)_{u} !(m-t)_{u} !} .
\end{aligned}
$$

Definition 3.1.2. DL, Section 3.4] The algebra $\Gamma(\mathfrak{g})$ is the $R$-subalgebra of $\check{U}_{q}(\mathfrak{g})$ generated by the elements 


$$
\begin{aligned}
K_{\alpha_{i}}^{-1} & (1 \leq i \leq n), \\
\left(\begin{array}{c}
K_{\alpha_{i}} ; 0 \\
t
\end{array}\right):=\prod_{s=1}^{t}\left(\frac{K_{\alpha_{i}} q_{i}^{-s+1}-1}{q_{i}^{s}-1}\right) & (t \geq 1,1 \leq i \leq n), \\
E_{i}^{(t)}:=\frac{E_{i}^{t}}{[t]_{q_{i}} !} & (t \geq 1,1 \leq i \leq n), \\
F_{i}^{(t)}:=\frac{F_{i}^{t}}{[t]_{q_{i}} !} & (t \geq 1,1 \leq i \leq n),
\end{aligned}
$$

where $q_{i}=q^{d_{i}}$ for $1 \leq i \leq n$.

Let $\mathcal{C}$ be the strictly full subcategory of $\Gamma(\mathfrak{g})$-mod whose objets are $\Gamma(\mathfrak{g})$ modules $M$ such that $M$ is a free $R$-module of finite rank with a basis in which the operators $K_{i}$ and $\left(\begin{array}{c}K_{i} ; 0 \\ t\end{array}\right)$ act by diagonal matrices with the eigenvalues $q_{i}^{m}$ and $\left(\begin{array}{c}m \\ t\end{array}\right)_{q_{i}}$ respectively.

Definition 3.1.3. DL, Section 4.1] Let $R_{q}[G]$ denote the $R$-submodule of $\operatorname{Hom}_{R}(\Gamma(\mathfrak{g}), R)$ spanned by the coordinate functions $t_{i}^{j}$ of representations $M$ from $\mathcal{C}$

$$
<g, t_{i}^{j}>=<g \cdot m_{i}, m^{j}>,
$$

where $\left(m_{i}\right)$ is a $R$-basis of $M,\left(m^{j}\right)$ is the dual basis of the dual module and $g \in \Gamma(\mathfrak{g})$. Since the subcategory $\mathcal{C}$ is a tensor one, $R_{q}[G]$ is a Hopf algebra.

Definition 3.1.4. DL, Section 6] The algebra $R_{q}[G] /\left[p_{\ell}(q) R_{q}[G]\right]$ is denoted by $\mathcal{O}_{\epsilon}(G)_{\mathbb{Q}(\epsilon)}$ and is called the quantized coordinate algebra of $G$ over $\mathbb{Q}(\epsilon)$ at the root of unity $\epsilon$. In the same way as for $\mathcal{O}_{\epsilon}(G)_{\mathbb{Q}(\epsilon)}$, we can form the $\mathbb{Q}(\epsilon)$-Hopf algebra $\Gamma_{\epsilon}(\mathfrak{g}):=\Gamma(\mathfrak{g}) /\left[p_{\ell}(q) \Gamma(\mathfrak{g})\right]$.

Using the following definition, we can relate the Hopf algebras $\mathcal{O}_{\epsilon}(G)_{\mathbb{Q}(\epsilon)}$ and $\Gamma_{\epsilon}(\mathfrak{g})$.

Definition 3.1.5. Let $U$ and $H$ be two Hopf algebras over a ring $A$. A Hopf pairing between them is a bilinear form $(-,-): H \times U \rightarrow A$ such that, for all $u, v \in U$ and $f, h \in H$,

(i) $(h, u v)=\left(h_{(1)}, u\right)\left(h_{(2)}, v\right)$;

(ii) $(f h, u)=\left(f, u_{(1)}\right)\left(h, u_{(2)}\right)$;

(iii) $(1, u)=\varepsilon(u)$ and $(h, 1)=\varepsilon(h)$;

(iv) $(h, \mathcal{S}(u))=(\mathcal{S}(h), u)$.

Given a Hopf pairing, the induced maps $U \rightarrow H^{*}$ and $H \rightarrow U^{*}$ actually map $U \rightarrow H^{\circ}$ and $H \rightarrow U^{\circ}$ and the later are Hopf algebra homomorphisms, where $H^{\circ}$ and $U^{\circ}$ are the Sweedler duals of $H$ and $U$ respectively. The pairing is called perfect or non-degenerate if the above maps are both injections. In our case, we have the following. 
Proposition 3.1.6. [DL, Lemmas 4.1 and 6.1]. There exists a perfect Hopf pairing $R_{q}[G] \otimes_{R} \Gamma(\mathfrak{g}) \rightarrow R$, which induces a perfect Hopf pairing $\mathcal{O}_{\epsilon}(G)_{\mathbb{Q}(\epsilon)} \otimes_{\mathbb{Q}(\epsilon)} \Gamma_{\epsilon}(\mathfrak{g}) \rightarrow \mathbb{Q}(\epsilon)$. In particular, $\mathcal{O}_{\epsilon}(G)_{\mathbb{Q}(\epsilon)} \subseteq \Gamma_{\epsilon}(\mathfrak{g})^{\circ}$ and $\Gamma_{\epsilon}(\mathfrak{g}) \subseteq$ $\mathcal{O}_{\epsilon}(G)_{\mathbb{Q}(\epsilon)}^{\circ}$

If $k$ is any field containing $\mathbb{Q}(\epsilon)$, one can obtain a $k$-form of $\mathcal{O}_{\epsilon}(G)_{\mathbb{Q}(\epsilon)}$, namely $\mathcal{O}_{\epsilon}(G)_{k}:=\mathcal{O}_{\epsilon}(G)_{\mathbb{Q}(\epsilon)} \otimes_{\mathbb{Q}(\epsilon)} k$. When $k=\mathbb{C}$ we simply write $\mathcal{O}_{\epsilon}(G)$ for $\mathcal{O}_{\epsilon}(G)_{\mathbb{C}}$. The following two results imply by Proposition 2.1.4 that $\mathcal{O}_{\epsilon}(G)$ is a central extension of $\mathcal{O}(G)$ by a finite-dimensional Hopf algebra.

Theorem 3.1.7. DL, Prop. 6.4 and Thm. 7.2].

(a) $\mathcal{O}_{\epsilon}(G)$ contains a central Hopf subalgebra isomorphic to the coordinate algebra of functions $\mathcal{O}(G)$ on $G$.

(b) $\mathcal{O}_{\epsilon}(G)$ is a projective $\mathcal{O}(G)$-module of rank $\ell^{\operatorname{dim} G}$.

Theorem 3.1.8. BG, Section III.7.11]. $\mathcal{O}_{\epsilon}(G)$ is a free $\mathcal{O}(G)$-module of rank $\ell^{\operatorname{dim} G}$.

3.2. A maximal torus. We show now that the inclusion given by Theorem 3.1.7 (a) determines a maximal torus $\mathbf{T}$ of $G$.

Let $k=\mathbb{C}$. In DL, Section 9.2], De Concini and Lyubashenko define an action of $\mathbb{C}^{n}$ on $\Gamma_{\epsilon}(\mathfrak{g})$ : Let $\phi^{\prime}: \Gamma(\mathfrak{g}) \rightarrow \Gamma_{\epsilon}(\mathfrak{g})$ be the canonical projection and consider the primitive elements given by

$$
H_{i}=\phi^{\prime}\left(\frac{K_{\alpha_{i}}^{l}-1}{l\left(q_{i}^{l}-1\right)}\right) \in \Gamma_{\epsilon}(\mathfrak{g}), \text { for all } 1 \leq i \leq n .
$$

Then for any $n$-tuple $\left(p_{1}, \ldots, p_{n}\right) \in \mathbb{C}^{n}$ and for any finite-dimensional $\Gamma_{\epsilon}(\mathfrak{g})$-module $M$ the elements $\exp \left(\sum_{i} p_{i} H_{i}\right)$ defines operators which commute with every $\Gamma_{\epsilon}(\mathfrak{g})$-module homomorphism. Hence, they define characters on $\mathcal{O}_{\epsilon}(G)$. Obviously, the elements $\exp \left(\sum_{i} p_{i} H_{i}\right) \in \mathcal{O}_{\epsilon}(G)^{*}$ form a group and the map given by

$$
\phi: \mathbb{C}^{n} \rightarrow \operatorname{Alg}\left(\mathcal{O}_{\epsilon}(G), k\right), \quad\left(p_{1}, \ldots, p_{n}\right) \mapsto \exp \left(\sum_{i} p_{i} H_{i}\right),
$$

defines a group homomorphism whose kernel is the subgroup $2 \pi i l \mathbb{Z}^{n}$.

Lemma 3.2.1. The map $(\mathbb{C} / 2 \pi i l \mathbb{Z})^{n} \hookrightarrow \operatorname{Alg}\left(\mathcal{O}_{\epsilon}(G), k\right)$ is an isomorphism.

Proof. See [DL, Thm. 10.8].

Furthermore, the inclusion $\mathcal{O}(G) \stackrel{\iota}{\rightarrow} \mathcal{O}_{\epsilon}(G)$ given by Theorem 3.1.7 $(a)$ induces by restriction a group homomorphism $\operatorname{Alg}\left(\mathcal{O}_{\epsilon}(G), k\right) \stackrel{{ }_{\iota}}{\longrightarrow} \operatorname{Alg}(\mathcal{O}(G), k)$. The composition of this group map with $\phi$ defines a group homomorphism

$$
\varphi: \mathbb{C}^{n} \stackrel{\phi}{\rightarrow} \operatorname{Alg}\left(\mathcal{O}_{\epsilon}(G), k\right) \stackrel{{ }^{\iota}}{\rightarrow} \operatorname{Alg}(\mathcal{O}(G), k)=G,
$$

whose kernel is the subgroup $2 \pi i \mathbb{Z}^{n}$, by $[\mathrm{DL}$, Prop. 9.3 (c)]. Let $\mathbf{T}$ be the subgroup of $G$ given by the image of $\varphi$. Clearly $\mathbf{T}$ is a maximal torus of $G$. 
3.3. An exact sequence. We end this section by spelling out explicitly the quotient of $\mathcal{O}_{\epsilon}(G)$ by its central Hopf subalgebra $\mathcal{O}(G)$.

Let $\overline{\mathcal{O}_{\epsilon}(G)}=\mathcal{O}_{\epsilon}(G) /\left[\mathcal{O}(G)^{+} \mathcal{O}_{\epsilon}(G)\right]$ and denote by $\pi: \mathcal{O}_{\epsilon}(G) \rightarrow \overline{\mathcal{O}_{\epsilon}(G)}$ the quotient map. By Theorems [3.1.7 and 3.1.8, $\overline{\mathcal{O}_{\epsilon}(G)}$ is a Hopf algebra of dimension $\ell^{\operatorname{dim} G}$. Since $\mathcal{O}_{\epsilon}(G)$ is a free $\mathcal{O}(G)$-module, it is in particular faithfully flat and by Proposition 2.1.4 it follows that $\mathcal{O}(G)=\mathcal{O}_{\epsilon}(G)^{\mathrm{co} \pi}=$ ${ }^{\text {co } \pi} \mathcal{O}_{\epsilon}(G)$. This implies that $\mathcal{O}_{\epsilon}(G)$ fits into the exact sequence

$$
1 \rightarrow \mathcal{O}(G) \rightarrow \mathcal{O}_{\epsilon}(G) \rightarrow \overline{\mathcal{O}_{\epsilon}(G)} \rightarrow 1 .
$$

We want now to determined the Hopf algebra quotient $\overline{\mathcal{O}_{\epsilon}(G)}$. Consider the quantized enveloping algebra $U_{q}(\mathfrak{g})$ with associated root system $\Phi$ and Weyl group $W$. The braid group $B_{W}$ of type $\Phi$ associated to the Weyl group $W$ is the group generated by the elements $t_{1}, \ldots, t_{n}$ satisfying the relations $t_{i} t_{j} \cdots=t_{j} t_{i} \cdots$, for $i \neq j$ with $m_{i j}$ factors on both sides, where $m_{i j}$ is the order of the product $s_{\alpha_{i}} s_{\alpha_{j}}$ of the simple reflections $s_{\alpha_{i}}$ and $s_{\alpha_{j}}$ in $W$. Lusztig, and independently Levendorskii and Soibelman, proved that the generators $t_{i}$ satisfy the braid relations and that $B_{W}$ acts via algebra automorphisms on $U_{q}(\mathfrak{g})$. For a detailed discussion see [J], Section 8].

Consider now the Hopf algebra $\check{U}_{\epsilon}(\mathfrak{g})$, which by a result due to De Concini, Kac and Procesi, has the same generators and the same relations as $\check{U}_{q}(\mathfrak{g})$ but with $q$ replaced by $\epsilon$ and admits an action of $B_{W}$, see [DK] and [DKP. Let $Z_{0}$ be the smallest $B_{W}$-invariant subalgebra of $\check{U}_{\epsilon}(\mathfrak{g})$ containing the elements

$$
K_{l \alpha}=K_{\alpha}^{l}, E_{i}^{l}, F_{i}^{l} \quad \text { for } \alpha \in P \text { and } 1 \leq i \leq n .
$$

Then by [BG, Thm. III.6.2], $Z_{0}$ is a central Hopf subalgebra of $\check{U}_{\epsilon}(\mathfrak{g})$ and $\check{U}_{\epsilon}(\mathfrak{g})$ is a free $Z_{0}$-module of rank $\ell^{\operatorname{dim} \mathfrak{g}}$. The Hopf algebra of dimension $\ell^{\operatorname{dim} \mathfrak{g}}$ given by the quotient $\mathbf{u}_{\epsilon}(\mathfrak{g}):=\check{U}_{\epsilon}(\mathfrak{g}) /\left[Z_{0}^{+} \check{U}_{\epsilon}(\mathfrak{g})\right]$ is called the restricted quantized enveloping algebra (or the Frobenius-Lusztig kernel) of $\mathfrak{g}$ at $\epsilon$.

Although it is well-known that the Hopf algebra $\overline{\mathcal{O}_{\epsilon}(G)}$ is isomorphic to the dual of the restricted quantized enveloping algebra $\mathbf{u}_{\epsilon}(\mathfrak{g})$, we could only find the following reference.

Theorem 3.3.1. The Hopf algebras $\overline{\mathcal{O}_{\epsilon}(G)}$ and $\mathbf{u}_{\epsilon}(\mathfrak{g})^{*}$ are isomorphic.

Proof. See [BG, Thm. III.7.10].

Remark 3.3.2. Many authors define the Frobenius-Lusztig kernel $\mathbf{u}_{\epsilon}(\mathfrak{g})$ as the subalgebra of $\Gamma_{\epsilon}(\mathfrak{g})$ generated by the elements $E_{i}, F_{i}$ and $K_{\alpha_{i}}$ for $1 \leq i \leq$ $n$, which is indeed a Hopf subalgebra of $\Gamma_{\epsilon}(\mathfrak{g})$. Nevertheless, this definition coincides with the one given here, see BG for details. By Proposition 3.1.6 we know that there exists a perfect pairing $\mathcal{O}_{\epsilon}(G) \otimes_{\mathbb{Q}(\epsilon)} \Gamma_{\epsilon}(\mathfrak{g}) \rightarrow \mathbb{Q}(\epsilon)$, which induces a perfect pairing $\overline{\mathcal{O}_{\epsilon}(G)} \otimes_{\mathbb{Q}(\epsilon)} \widehat{U}_{\epsilon}(\mathfrak{g}) \rightarrow \mathbb{Q}(\epsilon)$, where $\widehat{U}_{\epsilon}(\mathfrak{g})$ is the Hopf subalgebra of $\Gamma_{\epsilon}(\mathfrak{g})$ generated by the elements $E_{i}, F_{i}$ and $K_{\alpha_{i}}$ for $1 \leq i \leq n$. This therefore yields a Hopf algebra homomorphism from $\overline{\mathcal{O}_{\epsilon}(G)}$ to $\widehat{U}_{\epsilon}(\mathfrak{g})^{*}$ which is an isomorphism by BG, Proof of Thm. III.7.10]. Hence, $\mathbf{u}_{\epsilon}(\mathfrak{g})$ is 
isomorphic to $\widehat{U}_{\epsilon}(\mathfrak{g})$ and the epimorphism $\pi: \mathcal{O}_{\epsilon}(G) \rightarrow \overline{\mathcal{O}_{\epsilon}(G)}$ corresponds to an injection ${ }^{t} \pi: \mathbf{u}_{\epsilon}(\mathfrak{g}) \rightarrow \Gamma_{\epsilon}(\mathfrak{g}) \subseteq \mathcal{O}_{\epsilon}(G)^{\circ}$.

Summarizing, the quantized coordinate algebra $\mathcal{O}_{\epsilon}(G)$ of $G$ at $\epsilon$ is a central extension of $\mathcal{O}(G), \mathcal{O}_{\epsilon}(G)$ is faithfully flat over $\mathcal{O}(G)$ and fits into the exact sequence

$$
1 \rightarrow \mathcal{O}(G) \stackrel{\iota}{\rightarrow} \mathcal{O}_{\epsilon}(G) \stackrel{\pi}{\rightarrow} \mathbf{u}_{\epsilon}(\mathfrak{g})^{*} \rightarrow 1
$$

\section{Finite-dimensional Quotients of $\mathcal{O}_{\epsilon}(G)$}

We keep the notation of Section 3, in particular, we have a fixed torus $\mathbf{T}$ of $G$, see Subsection 3.2 In what follows we construct new examples of finite-dimensional Hopf algebras which are extensions of functions algebras of suitable finite subgroups $\Gamma$ of a connected, simply connected complex semisimple Lie group $G$ by the dual of the Frobenius-Lusztig kernel $\mathbf{u}_{\epsilon}(\mathfrak{g})$.

4.1. Constructions. We begin by explicitly stating the following classical fact.

Lemma 4.1.1. Let $\Gamma$ be a finite subgroup of $G$. Then there exists a Hopf algebra epimorphism $\varrho: \mathcal{O}(G) \rightarrow k^{\Gamma}$. Conversely, if $\varrho: \mathcal{O}(G) \rightarrow H$ is a Hopf algebra epimorphism with $H$ finite-dimensional, then there exist a finite subgroup $\Gamma$ of $G$ such that $H \simeq k^{\Gamma}$.

Now we perform the general constructions from Subsection 2.2 in the setting of $\mathcal{O}_{\epsilon}(G)$.

Construction 1. Let $\Gamma$ be a finite group and let $\sigma: \Gamma \rightarrow G$ be an embedding in $G$. Let $\varrho: \mathcal{O}(G) \rightarrow k^{\Gamma}$ be the epimorphism of Hopf algebras given by Lemma 4.1.1. Then the exact sequence of Hopf algebras (3.3.3) gives rise by Proposition 2.1.5 to an exact sequence of finite-dimensional Hopf algebras

$$
1 \rightarrow k^{\Gamma} \stackrel{j}{\rightarrow} \mathcal{O}_{\epsilon}(G) /(\mathcal{J}) \stackrel{\bar{\pi}}{\rightarrow} \mathbf{u}_{\epsilon}(\mathfrak{g})^{*} \rightarrow 1,
$$

where $\mathcal{J}=\operatorname{Ker} \varrho,(\mathcal{J})=\mathcal{O}_{\epsilon}(G) \mathcal{J}$ and $k^{\Gamma}$ is central in $\mathcal{O}_{\epsilon}(G) /(\mathcal{J})$. Thus, the Hopf algebra $\mathcal{O}_{\epsilon}(G) /(\mathcal{J})$ is given by a pushout and by Lemma 2.2.3 $\operatorname{dim} \mathcal{O}_{\epsilon}(G) /(\mathcal{J})=|\Gamma| \ell^{\operatorname{dim} \mathfrak{g}}$ is finite. From now on we write $A_{\sigma}=\mathcal{O}_{\epsilon}(G) /(\mathcal{J})$.

Construction 2. Let $\sigma$ be as in Construction 11 and $r: \mathbf{u}_{\epsilon}(\mathfrak{g})^{*} \rightarrow L$ be an epimorphism of Hopf algebras. Since by Theorem 2.1.3 the central extension $A_{\sigma}$ is cleft, there exists a retraction $\xi: A_{\sigma} \rightarrow k^{\Gamma}$ in $\operatorname{Reg}_{\varepsilon}\left(A_{\sigma}, k^{\Gamma}\right)$. Hence, by Proposition 2.2.7 we get an exact sequence of Hopf algebras associated with the triple $(\sigma, r, \xi)$ :

$$
1 \rightarrow K_{r, \xi} \rightarrow A_{\sigma, r, \xi} \rightarrow L \rightarrow 1
$$

where $K_{r, \xi}=k^{\Gamma} /\left(I \cap k^{\Gamma}\right)$ is central in $A_{\sigma, r, \xi}$ and $\operatorname{dim} A_{\sigma, r, \xi}$ is finite.

Remark 4.1.3. In [M2, Thm. 6.3], using the coradical filtration of $\mathbf{u}_{\epsilon}(\mathfrak{g})$, Müller classified all possible Hopf subalgebras of $\mathbf{u}_{\epsilon}(\mathfrak{g})$. Hence, all possible Hopf algebras epimorphisms $r: \mathbf{u}_{\epsilon}(\mathfrak{g})^{*} \rightarrow L$ are known. 
Although both constructions seem to be of interest, we concentrate on the first one to give new results concerning infinite families of finite-dimensional Hopf algebras. The study of the second one is left for future research.

The following lemma generalizes [M3, Prop 5.3].

Lemma 4.1.4. Consider the following commutative diagram of exact sequences of Hopf algebras

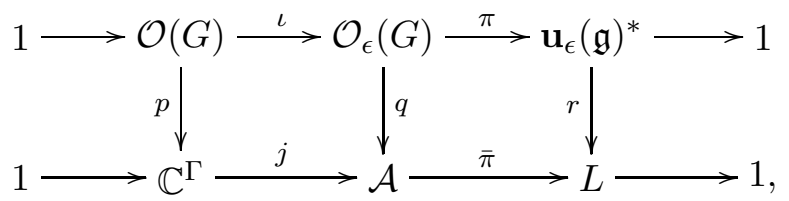

where $\Gamma$ is a finite group and the Hopf maps $p, q$ and $r$ are surjective. Let $\sigma: \Gamma \rightarrow G$ be the group monomorphism induced by $p$. Then

(a) The map $\mathbb{C}^{\Gamma} \stackrel{j}{\rightarrow} \mathcal{A}$ induces a group homomorphism $G\left(\mathcal{A}^{*}\right) \stackrel{{ }^{t} j}{\rightarrow} \Gamma$ and $\operatorname{Im} \sigma\left({ }^{t} j\right) \subseteq \mathbf{T} \cap \sigma(\Gamma)$.

(b) If $\mathcal{A}^{*}$ is pointed, then $\sigma(\Gamma)$ is a subgroup of the maximal torus $\mathbf{T}$.

Proof. (a) Since $L$ is a quotient of $\mathbf{u}_{\epsilon}(\mathfrak{g})^{*}$, which is finite-dimensional, $\operatorname{dim} L$ is finite. Moreover, as $\Gamma$ is finite by assumption, by Lemma $2.2 .3 \mathcal{A}$ is also finite-dimensional. Then, the exact sequence $1 \rightarrow \mathbb{C}^{\Gamma} \stackrel{j}{\rightarrow} \mathcal{A} \stackrel{\pi}{\rightarrow} L \rightarrow 1$ induces an exact sequence of Hopf algebras $1 \rightarrow L^{*} \stackrel{{ }^{t} \pi}{\rightarrow} \mathcal{A}^{*} \stackrel{{ }^{t} j}{\rightarrow} \mathbb{C}[\Gamma] \rightarrow 1$ and this, an exact sequence of groups

$$
1 \rightarrow G\left(L^{*}\right) \stackrel{{ }^{t} \pi}{\rightarrow} G\left(\mathcal{A}^{*}\right) \stackrel{{ }^{t} j}{\rightarrow} \Gamma,
$$

which fits into the commutative diagram of groups

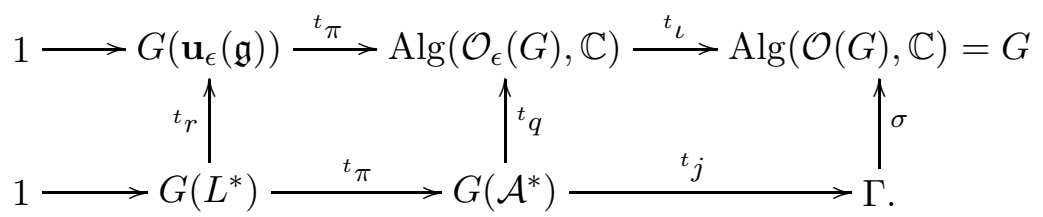

Since $q$ is surjective, it follows that ${ }^{t} q: G\left(\mathcal{A}^{*}\right) \rightarrow \operatorname{Alg}\left(\mathcal{O}_{\epsilon}(G), \mathbb{C}\right)$ is injective. But by Lemma 3.2.1, we know that $\operatorname{Alg}\left(\mathcal{O}_{\epsilon}(G), \mathbb{C}\right) \simeq(\mathbb{C} / 2 \pi i \ell \mathbb{Z})^{n}$ and by Subsection 3.2 the image of the restriction map ${ }^{t} \iota: \operatorname{Alg}\left(\mathcal{O}_{\epsilon}(G), \mathbb{C}\right) \rightarrow G$ is the maximal torus $\mathbf{T}$ of $G$. Hence the subgroup $\sigma\left({ }^{t} j\right)\left(G\left(\mathcal{A}^{*}\right)\right)$ of $\sigma(\Gamma)$ must be a subgroup of $\mathbf{T}$.

(b) Since ${ }^{t} j: \mathcal{A}^{*} \rightarrow \mathbb{C}[\Gamma]$ is surjective, by $[\mathrm{Mo}$, Cor. 5.3.5] the image of the coradical of $\mathcal{A}^{*}$ is the coradical of $\mathbb{C}[\Gamma]$. Hence, if $\mathcal{A}^{*}$ is pointed then ${ }^{t} j\left(G\left(\mathcal{A}^{*}\right)\right)=\Gamma$. Thus by $(a), \sigma(\Gamma)$ must be a subgroup of $\mathbf{T}$.

Using the following lemma we deduce properties of the $L$-extension $A$ of $\mathbb{C}^{\Gamma}$ from the properties of $\Gamma$ and $L$ and conversely.

Lemma 4.1.6. Let $1 \rightarrow \mathbb{C}^{\Gamma} \rightarrow A \rightarrow L \rightarrow 1$ be an exact sequence of finitedimensional Hopf algebras. Then 
(a) A is semisimple if and only if $L$ is semisimple.

(b) If $A$ is pointed, then $L$ is pointed. Moreover, let $p$ be an odd prime number and suppose that $|\Gamma|=p$ and $|G(L)| \leq p$. If $L$ is pointed, then $A$ is pointed.

Proof. (a) It is well-known that $A$ is semisimple if and only if $\mathbb{C}^{\Gamma}$ and $L$ are semisimple. Then the claim follows, since $\mathbb{C}^{\Gamma}$ is semisimple. (b) Since $L$ is a quotient of $A$, it follows from $[\mathrm{Mo}$, Cor. 5.3.5] that it is pointed if $A$ is pointed. The converse follows from [G1, Thm. 2.1].

Here is our first important result.

Theorem 4.1.7. Let $G$ be a connected, simply connected complex semisimple Lie group. Let $\Gamma$ be a finite group and let $\sigma: \Gamma \rightarrow G$ be an embedding of $\Gamma$ in $G$ such that $\sigma(\Gamma) \nsubseteq \mathbf{T}$. Then the Hopf algebra $A_{\sigma}$ constructed above is non-semisimple, non-pointed and its dual is also non-pointed. It is a quotient of $\mathcal{O}_{\epsilon}(G)$ of dimension $|\Gamma| \ell^{\operatorname{dim} \mathfrak{g}}$ and fits into the exact sequence

$$
1 \rightarrow \mathbb{C}^{\Gamma} \rightarrow A_{\sigma} \rightarrow \mathbf{u}_{\epsilon}(\mathfrak{g})^{*} \rightarrow 1,
$$

where $\mathbb{C}^{\Gamma}$ is central in $A_{\sigma}$.

Proof. We know that $\mathcal{O}_{\epsilon}(G)$ fits into the exact sequence (3.3.3) and it is a central $\mathbf{u}_{\epsilon}(\mathfrak{g})^{*}$-extension of $\mathcal{O}(G)$. Since $\sigma(\Gamma)$ is a subgroup of $G$, by Lemma 4.1 .1 there exists a Hopf algebra epimorphism $\varrho: \mathcal{O}(G) \rightarrow \mathbb{C}^{\Gamma}$. If we denote $\mathcal{J}=\operatorname{Ker} \varrho$ and $(\mathcal{J})=\mathcal{J O}_{\epsilon}(G)$, then $\mathbb{C}^{\Gamma} \simeq \mathcal{O}(G) / \mathcal{J}$ and by Proposition 2.1.5 the Hopf algebra $A_{\sigma}=\mathcal{O}_{\epsilon}(G) /(\mathcal{J})$ is given by a pushout diagram and fits into the exact sequence $1 \rightarrow \mathbb{C}^{\Gamma} \rightarrow A_{\sigma} \rightarrow \mathbf{u}_{\epsilon}(\mathfrak{g})^{*} \rightarrow 1$, where $\mathbb{C}^{\Gamma}$ is central in $A_{\sigma}$. By Lemma 4.1.6, $A_{\sigma}$ is non-semisimple and non-pointed since, as it is well-known, $\mathbf{u}_{\epsilon}(\mathfrak{g})$ is non-semisimple and non-pointed. The fact that $A_{\sigma}^{*}$ is non-pointed follows from Lemma 4.1.4.

4.2. Group cohomology. To describe the isomorphism classes of this type of extensions we shall need some basic facts from cohomology of groups. Let $G, \Gamma$ be two groups and suppose that there exists an action of $\Gamma$ on $G$, say $G \times \Gamma \leftrightharpoons G$, such that for all $g, h \in G$ and $x \in \Gamma$ we have that

$$
(g h)<x=(g \leftarrow x)(h \leftarrow x) \text { and } 1<x=1 .
$$

Given two groups $K$ and $L$, denote by $\operatorname{Map}(K, L)$ the set of maps from $K$ to $L$. For $n=0,1$ we define differential maps $\partial_{n}$ by

$$
\begin{array}{ll}
\partial_{0}: \operatorname{Map}(1, G) \rightarrow \operatorname{Map}(\Gamma, G), & \partial_{0}(g)(x)=(g<x) g^{-1}, \\
\partial_{1}: \operatorname{Map}(\Gamma, G) \rightarrow \operatorname{Map}(\Gamma \times \Gamma, G), & \partial_{1}(v)(x, y)=(v(x) \leftarrow y) v(y) v(x y)^{-1},
\end{array}
$$

for all $x, y \in \Gamma, g \in G$ and $v \in \operatorname{Map}(\Gamma, G)$. Note that $\operatorname{Map}(1, G)=G$. As usual, we have that $\partial^{2}=1$.

Definition 4.2.2. ( $i)$ A map $u \in \operatorname{Map}(\Gamma, G)$ is called a 1-coboundary if $u \in$ $\operatorname{Im} \partial_{0}$, that is, if there exists $g \in G$ such that $u(x)=\partial_{0}(g)(x)=(g \leftarrow x) g^{-1}$ for all $x \in \Gamma$. 
(ii) A map $v \in \operatorname{Map}(\Gamma, G)$ is called a 1-cocycle if $\partial_{1}(v)=1$, that is, if $v(x y)=(v(x)<y) v(y)$ for all $x, y \in \Gamma$.

Clearly, every 1-coboundary is a 1-cocycle. Denote by $Z^{1}(\Gamma, G)$ the subset of 1-cocycles in $\operatorname{Map}(\Gamma, G)$. Then $G=\operatorname{Map}(1, G)$ acts on $Z^{1}(\Gamma, G)$ via

$$
(g \cdot v)(x)=(g<x) v(x) g^{-1},
$$

for all $g \in G, v \in Z^{1}(\Gamma, G)$ and $x \in \Gamma$. We say that two 1-cocycles $v$ and $u$ are equivalent, denoted by $v \sim u$, if there exists $g \in G$ such that $v=g \cdot u$. Then we define:

$$
H^{1}(\Gamma, G):=Z^{1}(\Gamma, G) / G
$$

In particular, $\bar{v}=\overline{1}$ in $H^{1}(\Gamma, G)$ if and only if $v$ is a 1-coboundary.

Now we apply these notions in our setting. Let $G$ be a connected, simply connected, semisimple complex Lie group as in Section 3 and let $\sigma: \Gamma \rightarrow G$ be an embedding of a finite group $\Gamma$.

From now on we assume that $G$ is simple.

We begin by showing that the $\mathbf{u}_{\epsilon}(\mathfrak{g})^{*}$-extension $\mathcal{O}_{\epsilon}(G)$ of $\mathcal{O}(G)$ satisfies the property $(L)$ of Subsection 2.3 .

Lemma 4.2.4. Every automorphism of $\mathbf{u}_{\epsilon}(\mathfrak{g})$ induces an automorphism of $G$, which leaves invariant the torus $\mathbf{T}$.

Proof. By Remark 3.3.2, we can view $\mathbf{u}_{\epsilon}(\mathfrak{g})$ as a Hopf subalgebra of $\Gamma_{\epsilon}(\mathfrak{g})$ via the inclusion ${ }^{t} \pi: \mathbf{u}_{\epsilon}(\mathfrak{g}) \rightarrow \Gamma_{\epsilon}(\mathfrak{g})$. Let $\bar{F}$ be an automorphism of $\mathbf{u}_{\epsilon}(\mathfrak{g})$. By [M1, Cor. 5.10], there exists a unique automorphism $F$ of $\Gamma_{\epsilon}(\mathfrak{g})$ such that $\left.F\right|_{\mathbf{u}_{\epsilon}(\mathfrak{g})}=\bar{F}$, that is $F^{t} \pi={ }^{t} \pi \bar{F}$.

Consider now the quantum Frobenius map $\eta: \Gamma_{\epsilon}(\mathfrak{g}) \rightarrow U(\mathfrak{g})_{\mathbb{Q}(\epsilon)}$, defined on the generators of $\Gamma_{\epsilon}(\mathfrak{g})$ by

$$
\begin{gathered}
\eta\left(E_{i}^{(m)}\right)=\left\{\begin{array}{cc}
e_{i}^{(m / \ell)} & \text { if } \ell \mid m \\
0 & \text { otherwise }
\end{array} \quad, \quad \eta\left(F_{i}^{(m)}\right)=\left\{\begin{array}{cc}
f_{i}^{(m / \ell)} & \text { if } \ell \mid m \\
0 & \text { otherwise }
\end{array},\right.\right. \\
\eta\left(\left(\begin{array}{c}
K_{\alpha_{i}} ; 0 \\
m
\end{array}\right)\right)=\left\{\begin{array}{cc}
\left(\begin{array}{c}
h_{i} ; 0 \\
m
\end{array}\right) & \text { if } \ell \mid m \\
0 & \text { otherwise }
\end{array} \quad, \quad \eta\left(K_{\alpha_{i}}^{-1}\right)=1, \text { for all } 1 \leq i \leq n .\right.
\end{gathered}
$$

Then by [DL, Thm. 6.3], $\eta$ is a well-defined Hopf algebra map and its kernel is the two-sided ideal generated by the set

$$
\left\{E_{i}^{(m)}, F_{i}^{(m)},\left(\begin{array}{c}
K_{\alpha_{i}} ; 0 \\
m
\end{array}\right), K_{\alpha_{i}}-1, p_{\ell}(q) \mid m>0, \ell \nmid m\right\} .
$$

Using the explicit description of the automorphism group of $\mathbf{u}_{\epsilon}(\mathfrak{g})$ given in AS2, Thm. 7.2], one can see that $F \operatorname{Ker} \eta=\operatorname{Ker} \eta$. Hence $F$ factorizes through a Hopf algebra automorphism $\underline{F}: U(\mathfrak{g})_{\mathbb{Q}(\epsilon)} \rightarrow U(\mathfrak{g})_{\mathbb{Q}(\epsilon)}$. But by $\underline{\mathrm{H}}$ Thm. 3.1], we have that $U(\mathfrak{g})^{\circ} \simeq \mathcal{O}(G)$ (since $\mathfrak{g}$ is simple), and therefore the transpose ${ }^{t} \underline{F}$ of $\underline{F}$ induces an automorphism of $\mathcal{O}(G)$, obtaining in this way an automorphism $f$ of $G$ which comes from an automorphism $\underline{F}$ of $\mathbf{u}_{\epsilon}(\mathfrak{g})$. 
Finally, we show that $f(\mathbf{T})=\mathbf{T}={ }^{t} \iota\left(G_{\epsilon}\right)$, where $G_{\epsilon}=\operatorname{Alg}\left(\mathcal{O}_{\epsilon}(G), \mathbb{C}\right)$. Since by Proposition 3.1.6 there exists a perfect pairing between $\mathcal{O}_{\epsilon}(G)$ and $\Gamma_{\epsilon}(\mathfrak{g})$, the automorphism $F$ induces an automorphism ${ }^{t} F$ of $\mathcal{O}_{\epsilon}(G)$. Since $F$ factorizes through $\underline{F}$ we have that ${ }^{t} F \iota=\iota^{t} \underline{F}$ and hence,

$f(\mathbf{T})=f\left({ }^{t} \iota\left(G_{\epsilon}\right)\right)={ }^{t}\left(\iota{ }^{t} \underline{F}\right)\left(G_{\epsilon}\right)={ }^{t}\left({ }^{t} F \iota\right)\left(G_{\epsilon}\right)={ }^{t} \iota\left(F\left(G_{\epsilon}\right)\right)={ }^{t} \iota\left(G_{\epsilon}\right) \subseteq \mathbf{T}$.

Thus $f(\mathbf{T})=\mathbf{T}$, because $f$ is an automorphism.

Corollary 4.2.5. The $\mathbf{u}_{\epsilon}(\mathfrak{g})^{*}$-extension $\mathcal{O}_{\epsilon}(G)$ of $\mathcal{O}(G)$ satisfies $(L)$.

Proof. Since $\operatorname{dim} \mathbf{u}_{\epsilon}(\mathfrak{g})<\infty$, every automorphism $\alpha$ of $\mathbf{u}_{\epsilon}(\mathfrak{g})^{*}$ corresponds to an automorphism $\bar{F}$ of $\mathbf{u}_{\epsilon}(\mathfrak{g})$. Thus, from the proof of the lemma above, $\bar{F}$ induces an automorphism $F$ of $\Gamma_{\epsilon}(\mathfrak{g})$ such that $F^{t} \pi={ }^{t} \pi \bar{F}$. Hence ${ }^{t} F \in$ Aut $\mathcal{O}_{\epsilon}(G)$ and $\alpha \pi=\pi{ }^{t} F$, which implies the claim.

Definition 4.2.6. Denote by qAut $(G)$ the subgroup of $\operatorname{Aut}(G)$ generated by the automorphisms of $G$ coming from automorphisms of $\mathbf{u}_{\epsilon}(\mathfrak{g})$.

We fix now a Borel subgroup $B$ of $G$ which contains $\mathbf{T}$, which amounts to fixing a base $\Delta$ of the root system $\Phi$ determined by $\mathbf{T}$. Let $D$ be the subgroup of $\operatorname{Aut}(G)$ given by

$$
D=\{f \in \operatorname{Aut}(G) \mid f(\mathbf{T})=\mathbf{T} \text { and } f(B)=B\} .
$$

Each $f \in D$ induces an automorphism $\hat{f}$ of $\Phi$, since $f(\mathbf{T})=\mathbf{T}$. Moreover, since $f(B)=B, f$ preserves $\Delta$. Therefore, $\hat{f}$ belongs to the group of diagram automorphisms of $\Phi$. Furthermore, if $\operatorname{Int}(G)$ denotes the subgroup of inner automorphisms of $G$, then by [Hu2, Thm 27.4], $\operatorname{Int}(G)$ is normal in $\operatorname{Aut}(G)$ and $\operatorname{Aut}(G)=\operatorname{Int}(G) \rtimes D$, and $\operatorname{Int}(G)$ has finite index in $\operatorname{Aut}(G)$. Since for all $t \in \mathbf{T}$, the inner automorphism $\operatorname{Int}(t)$ of $G$ given by the conjugation fixes $\mathbf{T}$ and $B$, see [Hu2, Lemma 24.1], we see that the image $\operatorname{Int}(\mathbf{T})$ of $\mathbf{T}$ in $\operatorname{Aut}(G)$ is a subgroup of $D$.

On the other hand, it follows from [M1, Cor. 5.7] that $D \subseteq$ qAut $(G)$. Denote by $\operatorname{Int}\left(N_{G}(\mathbf{T})\right)$ the subgroup of inner automorphisms of $\operatorname{Aut}(G)$ given by the conjugation of elements in the normalizer $N_{G}(\mathbf{T})$ of $\mathbf{T}$ in $G$.

Lemma 4.2.7. (a) qAut $(G)$ is a subgroup of $\operatorname{Int}\left(N_{G}(\mathbf{T})\right) \rtimes D$.

(b) $\mathbf{T}$ acts on qAut $(G)$ by left multiplication of $\operatorname{Int}(\mathbf{T})$.

(c) The set $\mathrm{qAut}(G) / \mathbf{T}$ of orbits of the preceding action is finite.

Proof. Let $f \in \operatorname{qAut}(G)$. Then there exist $\alpha \in \operatorname{Int}(G), \beta \in D$ such that $f=\alpha \beta$. Since $f(\mathbf{T})=\mathbf{T}$ and $\beta(\mathbf{T})=\mathbf{T}$, it follows that $\alpha(\mathbf{T})=\mathbf{T}$. Hence $\alpha=\operatorname{Int}(g)$, for some $g \in N_{G}(\mathbf{T})$ and the claim $(a)$ follows.

Since $\operatorname{Int}(\mathbf{T}) \subseteq D \subseteq \mathrm{qAut}(G)$, the left multiplication by elements of $\operatorname{Int}(\mathbf{T})$ defines an action of $\mathbf{T}$ on qAut $(G)$.

Since by $(a)$, qAut $(G) \subseteq \operatorname{Int}\left(N_{G}(\mathbf{T})\right) \rtimes D$, it follows that

$$
|\operatorname{qAut}(G) / \mathbf{T}| \leq\left|\left[\operatorname{Int}\left(N_{G}(\mathbf{T})\right) \rtimes D\right] / \mathbf{T}\right| \leq\left|N_{G}(\mathbf{T}) / \mathbf{T}\right||D|=\left|W_{\mathbf{T}}\right||D|,
$$

where $W_{\mathbf{T}}=N_{G}(\mathbf{T}) / C_{G}(\mathbf{T})=N_{G}(\mathbf{T}) / \mathbf{T}$ is the Weyl group associated to $\mathbf{T}$. The claim follows since the orders of $W_{\mathbf{T}}$ and $D$ are finite. 
Let $\sigma: \Gamma \rightarrow G$ be an embedding of the finite group $\Gamma$. For any $f \in \operatorname{Aut}(G)$ we define an action of $\Gamma$ on $G$, depending on $\sigma$ and $f$, via the conjugation:

$$
G \times \Gamma \leftrightarrows G, \quad g<x=(f \sigma(x))^{-1} g(f \sigma(x)),
$$

for all $g \in G, x \in \Gamma$. Clearly, both conditions in (4.2.1) are satisfied. Hence, $u \in \operatorname{Map}(\Gamma, G)$ is a 1-coboundary if and only if there exists $g \in G$ such that

$$
u(x)=\partial_{0}(g)(x)=(g \leftarrow x) g^{-1}=(f \sigma(x))^{-1} g(f \sigma(x)) g^{-1},
$$

for all $x \in \Gamma$, and a map $v \in \operatorname{Map}(\Gamma, G)$ is a 1-cocycle if and only if

$$
v(x y)=(v(x)<y) v(y)=(f \sigma(y))^{-1} v(x)(f \sigma(y)) v(y),
$$

for all $x, y \in \Gamma$. As the action depends on $\sigma$ and $f$, we denote by $Z_{f, \sigma}^{1}(\Gamma, G)$ the set of 1-cocycles associated to this action.

Denote by $\operatorname{Emb}(\Gamma, G)$ the set of embeddings of $\Gamma$ in $G$.

Definition 4.2.11. Let $\Gamma$ be a finite group and $\sigma_{1}, \sigma_{2} \in \operatorname{Emb}(\Gamma, G)$. We say that $\sigma_{1}$ is equivalent to $\sigma_{2}$, denoted by $\sigma_{1} \sim \sigma_{2}$, if there exist $\tau \in \operatorname{Aut}(\Gamma)$, $f \in \operatorname{qAut}(G)$ and $v \in \operatorname{Map}(\Gamma, G)$ such that

$$
\sigma_{1}(\tau(x))=f\left(\sigma_{2}(x)\right) v(x) \text { for all } x \in \Gamma .
$$

Note that the map $v$ is uniquely determined by $f \sigma_{2}$ and $\sigma_{1} \tau$ with $v(x)=$ $f\left(\sigma_{2}(x)\right)^{-1} \sigma_{1}(\tau(x))$ for all $x \in \Gamma$. A straightforward computation shows that $v \in Z_{f, \sigma_{2}}^{1}(\Gamma, G)$.

Remark 4.2.12. If the 1-cocycle $v$ is a 1-coboundary, then there exists $g \in G$ such that $v(x)=\partial(g)(x)=(g<x) g^{-1}=f\left(\sigma_{2}(x)\right)^{-1} g f\left(\sigma_{2}(x)\right) g^{-1}$ for all $x \in \Gamma$ and this implies that

$$
\sigma_{1}(\tau(x))=g f\left(\sigma_{2}(x)\right) g^{-1} .
$$

That is, $\sigma_{1}$ can be obtained by the automorphism $\tau, f$ and the conjugation by an element of $G$.

Lemma 4.2.13. $\sim$ is an equivalence relation in $\operatorname{Emb}(\Gamma, G)$.

Proof. We show that $\sim$ is $(a)$ reflexive, $(b)$ symmetric and $(c)$ transitive.

(a) follows taking $f=\mathrm{id}, \tau=\mathrm{id}$ and $v=1$.

(b) Suppose that $\sigma_{1} \sim \sigma_{2}$. Then there exist $\tau \in \operatorname{Aut}(\Gamma), f \in \operatorname{qAut}(G)$ and $v \in Z_{f, \sigma_{2}}^{1}(\Gamma, G)$ such that (4.2.11) holds. Hence

$$
\sigma_{2}\left(\tau^{-1}(x)\right)=f^{-1}\left(\sigma_{1}(x)\right) f^{-1}\left(v\left(\tau^{-1}(x)\right)^{-1}\right)=f^{-1}\left(\sigma_{1}(x)\right) \hat{v}(x),
$$

for all $x \in \Gamma$, where $\hat{v}$ is given by $\hat{v}(x)=f^{-1}\left(v\left(\tau^{-1}(x)\right)^{-1}\right)$. Thus $\sigma_{2} \sim \sigma_{1}$.

(c) Let $\sigma_{1}, \sigma_{2}, \sigma_{3} \in \operatorname{Emb}(\Gamma, G)$ and suppose that $\sigma_{1} \sim \sigma_{2}$ and $\sigma_{2} \sim \sigma_{3}$, that is, there exist $f, g \in \operatorname{qAut}(G), \tau, t \in \operatorname{Aut}(\Gamma)$ and $v \in Z_{f, \sigma_{2}}^{1}(\Gamma, G)$, 
$u \in Z_{g, \sigma_{3}}^{1}(\Gamma, G)$ such that $\sigma_{1}(\tau(x))=f \sigma_{2}(x) v(x)$ and $\sigma_{2}(t(x))=g \sigma_{3}(x) u(x)$, for all $x \in \Gamma$. Using both equations we get that

$$
\begin{aligned}
\sigma_{1}(\tau(x)) & =f\left(g\left(\sigma_{3}\left(t^{-1}(x)\right)\right) u\left(t^{-1}(x)\right)\right) v(x) \\
& =f\left(g\left(\sigma_{3}\left(t^{-1}(x)\right)\right)\right) f\left(u\left(t^{-1}(x)\right)\right) v(x),
\end{aligned}
$$

and hence $\sigma_{1}(\tau t(x))=f\left(g\left(\sigma_{3}(x)\right)\right) f(u(x)) v(t(x))=f\left(g\left(\sigma_{3}(x)\right)\right) \hat{v}(x)$, where $\hat{v}(x)=f(u(x)) v(t(x))$ for all $x \in \Gamma$. It follows that $\sigma_{1} \sim \sigma_{3}$.

Definition 4.2.14. Let $\sigma \in \operatorname{Emb}(\Gamma, G)$ and $f \in \operatorname{qAut}(G)$. Then define $\mathbf{T}^{f \sigma}$ to be the subgroup of $\mathbf{T}$ given by

$$
\mathbf{T}^{f \sigma}=\bigcap_{y \in \Gamma} f \sigma(y) \mathbf{T} f \sigma\left(y^{-1}\right) .
$$

For any $\sigma \in \operatorname{Emb}(\Gamma, G)$ and $f \in \operatorname{qAut}(G)$, the subgroup $\mathbf{T}^{f \sigma}$ is stable under the action of $\Gamma$ on $G$ defined by $g<x=f \sigma\left(x^{-1}\right) g f \sigma(x)$ for all $x \in \Gamma$ and $g \in G$. We will see in Theorem 4.3.2 that the cocycle $v$ arising in (4.2.11) actually belongs to $Z_{f, \sigma}^{1}\left(\Gamma, \mathbf{T}^{f \sigma}\right)$.

Lemma 4.2.15. $Z_{f, \sigma}^{1}\left(\Gamma, \mathbf{T}^{f \sigma}\right)=Z_{(t \cdot f), \sigma}^{1}\left(\Gamma, \mathbf{T}^{(t \cdot f) \sigma}\right)$ for all $t \in \mathbf{T}$.

Proof. We first claim that $\mathbf{T}^{(t \cdot f) \sigma}=\mathbf{T}^{f \sigma}$ for all $t \in \mathbf{T}$. Indeed,

$$
\begin{aligned}
\mathbf{T}^{(t \cdot f) \sigma} & =\bigcap_{y \in \Gamma}(t \cdot f) \sigma(y) \mathbf{T}(t \cdot f) \sigma\left(y^{-1}\right)=\bigcap_{y \in \Gamma} t f \sigma(y) t^{-1} \mathbf{T} t f \sigma\left(y^{-1}\right) t^{-1} \\
& =\bigcap_{y \in \Gamma} t f \sigma(y) \mathbf{T} f \sigma\left(y^{-1}\right) t^{-1}=t \mathbf{T}^{f \sigma} t^{-1}=\mathbf{T}^{f \sigma}
\end{aligned}
$$

since $\mathbf{T}^{f \sigma} \subset \mathbf{T}$ and $t \in \mathbf{T}$. Suppose that $v \in Z_{(t \cdot f), \sigma}^{1}\left(\Gamma, \mathbf{T}^{(t \cdot f) \sigma}\right)$. Then $v(x) \in \mathbf{T}^{(t \cdot f) \sigma}=\mathbf{T}^{f \sigma}$ for all $x \in \Gamma$. Now we show that $v$ is a 1-cocycle with respect to $f$ and $\sigma$ :

$$
\begin{aligned}
v(x y) & =(t \cdot f)\left(\sigma\left(y^{-1}\right)\right) v(x)(t \cdot f)(\sigma(y)) v(y) \\
& =t f\left(\sigma\left(y^{-1}\right)\right) t^{-1} v(x) t f(\sigma(y)) t^{-1} v(y) \\
& =t f\left(\sigma\left(y^{-1}\right)\right) v(x) f(\sigma(y)) v(y) t^{-1} \\
& =f\left(\sigma\left(y^{-1}\right)\right) v(x) f(\sigma(y)) v(y) .
\end{aligned}
$$

The last equality follows from the fact that $f\left(\sigma\left(y^{-1}\right)\right) v(x) f(\sigma(y)) v(y) \in \mathbf{T}$ for all $x, y \in \Gamma$, by Definition 4.2.14. The other inclusion follows directly from similar computations.

A key step in the proof of our main result is the next lemma.

Lemma 4.2.16. Fix $\sigma \in \operatorname{Emb}(\Gamma, G), \tau \in \operatorname{Aut}(\Gamma)$ and $f \in \operatorname{qAut}(G)$. Define $\mathfrak{C}_{\sigma, f, \tau}=\left\{\eta \in \operatorname{Emb}(\Gamma, G) \mid \sigma \sim \eta\right.$ via the triple $\left.(\tau, f, v), v \in Z_{f, \sigma}^{1}\left(\Gamma, \mathbf{T}^{f \sigma}\right)\right\}$. 
Then $\mathbf{T}^{f \sigma}$ acts on $\mathfrak{C}_{\sigma, f, \tau}$ by $t \cdot \eta=\operatorname{Int}(t) \eta$ for all $\eta \in \mathfrak{C}_{\sigma, f, \tau}$. Moreover, there exists an injective map

$$
\mathfrak{C}_{\sigma, f, \tau} / \mathbf{T}^{f \sigma} \rightarrow H_{f, \sigma}^{1}\left(\Gamma, \mathbf{T}^{f \sigma}\right), \quad[\eta] \mapsto\left[v_{\eta}\right]
$$

where $v_{\eta}(x)=f(\sigma(x))^{-1} \eta(\tau(x))$ for all $x \in \Gamma$, is the unique 1-cocycle such that $\eta(\tau(x))=f(\sigma(x)) v_{\eta}(x)$ for all $x \in \Gamma$.

Proof. Let $\eta \in \mathfrak{C}_{\sigma, f, \tau}$. By definition there exists $v=v_{\eta} \in Z_{f, \sigma}^{1}\left(\Gamma, \mathbf{T}^{f \sigma}\right)$ such that $\eta(\tau(x))=f(\sigma(x)) v(x)$ for all $x \in \Gamma$. Then for all $t \in \mathbf{T}^{f \sigma}$ we have

$$
\begin{aligned}
t \cdot \eta(\tau(x)) & =t \eta(\tau(x)) t^{-1}=t f(\sigma(x)) v(x) t^{-1} \\
& =f(\sigma(x)) f(\sigma(x))^{-1} t f(\sigma(x)) v(x) t^{-1} \\
& =f(\sigma(x))\left[f(\sigma(x))^{-1} t f(\sigma(x))\right] v(x) t^{-1} \\
& =f(\sigma(x))(t<x) v(x) t^{-1} \\
& =f(\sigma(x))(t \cdot v)(x),
\end{aligned}
$$

which implies that $t \cdot \eta \in \mathfrak{C}_{\sigma, f, \tau}$, and that $\nu=t \cdot \eta$ if and only if $v_{\nu}=t \cdot v_{\eta}$ for all $t \in \mathbf{T}^{f \sigma}$. Hence the map $(t, \eta) \mapsto t \cdot \eta$ defines an action of $\mathbf{T}^{f \sigma}$ on $\mathfrak{C}_{\sigma, f, \tau}$. Let $[\eta]$ denote the class of $\eta$ in $\mathfrak{C}_{\sigma, f, \tau} / \mathbf{T}^{f \sigma}$. Then $[\eta]=[\nu]$ if and only if there exists $t \in \mathbf{T}^{f \sigma}$ such that $t \cdot \eta=\nu$. Thus, the map defined by

$$
\mathfrak{C}_{\sigma, f, \tau} / \mathbf{T}^{f \sigma} \rightarrow H_{f, \sigma}^{1}\left(\Gamma, \mathbf{T}^{f \sigma}\right), \quad[\eta] \mapsto\left[v_{\eta}\right],
$$

is well-defined and injective.

Recall that an algebraic group $G$ is a $d$-group if the coordinate $\operatorname{ring} \mathcal{O}(G)$ has a basis consisting of characters. Clearly any torus $T$ is a $d$-group.

Lemma 4.2.17. $\mathrm{Hu}$, Prop. 16.1 and Thm. 16.2]

(a) If $H$ is a closed subgroup of a d-group $G$, then $H$ is also a d-group.

(b) A connected d-group is a torus.

The following lemma is also crucial for the proof of our last theorem.

Lemma 4.2.18. The group $H_{f, \sigma}^{1}\left(\Gamma, \mathbf{T}^{f \sigma}\right)$ is finite.

Proof. Let $\mathbf{T}_{0}^{f \sigma}$ be the connected component of $\mathbf{T}^{f \sigma}$ which contains the identity and let $\mathfrak{T}=\mathbf{T}^{f \sigma} / \mathbf{T}_{0}^{f \sigma}$; in particular $|\mathfrak{T}|$ is finite. Since $\mathbf{T}$ is closed, it follows that $f(\sigma(x)) \mathbf{T} f\left(\sigma\left(x^{-1}\right)\right)$ is closed for all $x \in \Gamma$. Thus $\mathbf{T}^{f \sigma}$ is also closed. Then by Lemma4.2.17 $(a), \mathbf{T}^{f \sigma}$ and consequently $\mathbf{T}_{0}^{f \sigma}$ are $d$-groups. Since $\mathbf{T}_{0}^{f \sigma}$ is connected, it follows from Lemma 4.2.17 (b) that $\mathbf{T}_{0}^{f \sigma}$ is a torus and consequently $\mathbf{T}_{0}^{f \sigma}$ is a divisible group.

As the action of $\Gamma$ on $\mathbf{T}^{f \sigma}$ is given by the conjugation via $f \sigma$, every $x \in \Gamma$ acts on $\mathbf{T}^{f \sigma}$ by a continuous automorphism. Hence, $\mathbf{T}_{0}^{f \sigma}$ is stable under the action of $\Gamma$ and whence the action of $\Gamma$ on $\mathbf{T}^{f \sigma}$ induces an action of $\Gamma$ on $\mathfrak{T}$. Thus we have an exact sequence of $\Gamma$-modules

$$
\mathbf{T}_{0}^{f \sigma \stackrel{\alpha}{\longrightarrow}} \mathbf{T}^{f \sigma \stackrel{\beta}{\longrightarrow}} \mathfrak{T},
$$


which by $[\mathrm{Br}$, Prop. III.6.1] induces the long exact sequence

$$
\begin{aligned}
& 0 \longrightarrow H_{f, \sigma}^{0}\left(\Gamma, \mathbf{T}_{0}^{f \sigma}\right) \stackrel{\alpha_{*}^{0}}{\longrightarrow} H_{f, \sigma}^{0}\left(\Gamma, \mathbf{T}^{f \sigma}\right) \stackrel{\beta_{*}^{0}}{\longrightarrow} H_{f, \sigma}^{0}(\Gamma, \mathfrak{T}) \longrightarrow \\
& \longrightarrow H_{f, \sigma}^{1}\left(\Gamma, \mathbf{T}_{0}^{f \sigma}\right) \stackrel{\alpha_{*}^{1}}{\longrightarrow} H_{f, \sigma}^{1}\left(\Gamma, \mathbf{T}^{f \sigma}\right) \stackrel{\beta_{*}^{1}}{\longrightarrow} H_{f, \sigma}^{1}(\Gamma, \mathfrak{T}) \longrightarrow \cdots
\end{aligned}
$$

Since $\Gamma$ and $\mathfrak{T}$ are finite groups, the order of $H_{f, \sigma}^{1}(\Gamma, \mathfrak{T})$ is finite. Hence, it is enough to show that $\left|H_{f, \sigma}^{1}\left(\Gamma, \mathbf{T}_{0}^{f \sigma}\right)\right|$ is finite, because in such a case $\left|H_{f, \sigma}^{1}\left(\Gamma, \mathbf{T}^{f \sigma}\right)\right|=\left|\operatorname{Im} \alpha_{*}^{1}\right|\left|\operatorname{Im} \beta_{*}^{1}\right|$ is also finite.

By $\left[\mathrm{Br}\right.$, Cor. III.10.2], we know that $H_{f, \sigma}^{n}\left(\Gamma, \mathbf{T}_{0}^{f \sigma}\right)$ is annihilated by $m=$ $|\Gamma|$ for all $n>0$. Then, for all $\alpha \in Z_{f, \sigma}^{1}\left(\Gamma, \mathbf{T}_{0}^{f \sigma}\right)$ there exists $t \in \mathbf{T}_{0}^{f \sigma}$ such that $\alpha^{m}=\partial(t)$. Since $\mathbf{T}_{0}^{f \sigma}$ is divisible, there exists $s \in \mathbf{T}_{0}^{f \sigma}$ such that $t=s^{m}$. Let $\beta=\partial\left(s^{-1}\right) \alpha$, then $\beta^{m}=1$ and therefore $\beta \in Z_{f, \sigma}^{1}\left(\Gamma, D_{m}\right)$, where $D_{m}=\left\{t \in \mathbf{T}_{0}^{f \sigma} \mid t^{m}=1\right\}$. Moreover, $[\alpha]=[\beta]$ and the inclusion of the set of 1-cocycles defines a surjective map $H_{f, \sigma}^{1}\left(\Gamma, D_{m}\right) \rightarrow H_{f, \sigma}^{1}\left(\Gamma, \mathbf{T}_{0}^{f \sigma}\right)$. As $\mathbf{T}_{0}^{f \sigma}$ is a torus, $D_{m}$ is a finite group and consequently $\left|H_{f, \sigma}^{1}\left(\Gamma, D_{m}\right)\right|$ is finite, which implies that $\left|H_{f, \sigma}^{1}\left(\Gamma, \mathbf{T}_{0}^{f \sigma}\right)\right|$ is also finite.

4.3. Isomorphisms. In this subsection we study the isomorphisms between the Hopf algebras $A_{\sigma}$ for a fixed finite group $\Gamma$. In particular, we prove in Theorem 4.3.5 that under certain conditions, there exists an infinite family of pairwise non-isomorphic finite-dimensional Hopf algebras which are nonsemisimple, non-pointed and their duals are also non-pointed.

Fix $\Gamma$ a finite group; let $\sigma_{1}, \sigma_{2} \in \operatorname{Emb}(\Gamma, G)$ and denote $A_{i}=A_{\sigma_{i}}$. By Corollary 4.2.5 we know that every automorphism of $\mathbf{u}_{\epsilon}(\mathfrak{g})^{*}$ can be lifted to an automorphism of $\mathcal{O}(G)$, that is, the $\mathbf{u}_{\epsilon}(\mathfrak{g})^{*}$-extension $\mathcal{O}_{\epsilon}(G)$ of $\mathcal{O}(G)$ satisfies the property $(L)$. Moreover, the Frobenius-Lusztig kernels $\mathbf{u}_{\epsilon}(\mathfrak{g})$ are simple Hopf algebras, i.e. they have no non-trivial normal Hopf subalgebras, if the order $\ell$ of the root of unity $\epsilon$ and the determinant of the matrix $D C$ are relatively prime, by AS1, Prop. A.3]. Here $C=\left(a_{i j}\right)$ is the Cartan matrix of $\mathfrak{g}$ and $D$ is an invertible diagonal matrix such that $D C=C D$ and its non-zero entries are 1,2 or 3 . If $\mathfrak{g}$ is not of type $A_{n}$, this is always the case. Thus, in this situation $\mathcal{Z}\left(\mathbf{u}_{\epsilon}(\mathfrak{g})^{*}\right)=\mathbb{C}$, since otherwise $\mathbf{u}_{\epsilon}(\mathfrak{g})^{*}$ would contain a central proper Hopf subalgebra $\mathbf{v}$ and thus $\mathbf{u}_{\epsilon}(\mathfrak{g})^{*}$ is an extension of $\mathbf{v}$ by $\mathbf{u}_{\epsilon}(\mathfrak{g})^{*} / \mathbf{v}^{+} \mathbf{u}_{\epsilon}(\mathfrak{g})^{*}$, implying by duality that $\mathbf{u}_{\epsilon}(\mathfrak{g})$ contains a proper normal Hopf subalgebra dual to $\mathbf{u}_{\epsilon}(\mathfrak{g})^{*} / \mathbf{v}^{+} \mathbf{u}_{\epsilon}(\mathfrak{g})^{*}$.

Hence, $\mathbf{u}_{\epsilon}(\mathfrak{g})^{*}$ satisfies the property $(Z)$ if $\ell$ and $\operatorname{det} D C$ are relatively prime. For this reason, we assume from now on that

$$
\ell \text { and } \operatorname{det} D C \text { are relatively prime. }
$$

Recall that the equivalence $\sim$ (Definition 4.2.11) is given by $(\tau, f, v)$ where (i) $\tau \in \operatorname{Aut}(\Gamma)$, 
(ii) $f \in \operatorname{qAut}(G)$ and

(iii) $v \in Z_{f, \sigma_{2}}^{1}(\Gamma, G)$ with $v(1)=1$.

Now we are able to apply Theorem [2.3.6, which in this case gives:

Theorem 4.3.2. If $A_{1}$ and $A_{2}$ are isomorphic then $\sigma_{1} \sim \sigma_{2}$ via a triple $(\tau, f, v)$ with $v \in Z_{f, \sigma_{2}}^{1}\left(\Gamma, \mathbf{T}^{f \sigma_{2}}\right)$.

Proof. By Theorem 2.3.6, we know that the Hopf algebras $A_{1}$ and $A_{2}$ are isomorphic if and only if there exists a triple $(\underline{\omega}, g, u)$ such that $\underline{\omega} \in \operatorname{Aut}\left(\mathbb{C}^{\Gamma}\right)$, $g \in \mathrm{qAut}(\mathcal{O}(G))$, and $u \in \operatorname{Reg}_{1, \varepsilon}\left(\mathcal{O}_{\epsilon}(G), \mathbb{C}^{\Gamma}\right)$ is an algebra map which satisfies properties (2.3.7) and (2.3.8). The transposes of these maps induce the maps ${ }^{t} \underline{\omega}=\tau \in \operatorname{Aut}(\Gamma),{ }^{t} g=f \in \operatorname{qAut}(G)$ and ${ }^{t} u=\mu \in \operatorname{Map}\left(\Gamma, G_{\epsilon}\right)$, where $G_{\epsilon}=\operatorname{Alg}\left(\mathcal{O}_{\epsilon}(G), \mathbb{C}\right)$ and $\mu(1)=1$.

Let $v={ }^{t} \iota \mu$, then $v$ is a 1-cocycle which satisfies (iii): By Subsection 3.2 the image of ${ }^{t} \iota: G_{\epsilon} \rightarrow G$ is the maximal torus $\mathbf{T}$, thus $v={ }^{t} \iota \mu$ is a map $v: \Gamma \rightarrow \mathbf{T} \subset G$, which by equations (2.3.7) and (2.3.8) satisfies that

$$
\begin{aligned}
\sigma_{1}(\tau(x)) & =f\left(\sigma_{2}(x)\right) v(x), \text { and } \\
v(x y) & =f\left(\sigma_{2}(y)\right)^{-1} v(x) \sigma_{1}(\tau(y)) \\
& =f\left(\sigma_{2}(y)\right)^{-1} v(x) f\left(\sigma_{2}(y)\right) v(y) \\
& =(v(x) \leftarrow y) v(y) .
\end{aligned}
$$

for all $g, h \in \Gamma$. Moreover, from the equalities above it follows that

$$
v(x y) v(y)^{-1}=f\left(\sigma_{2}(y)\right)^{-1} v(x) f\left(\sigma_{2}(y)\right) \text { for all } x, y \in \Gamma,
$$

which implies that $f\left(\sigma_{2}(y)\right)^{-1} v(x) f\left(\sigma_{2}(y)\right) \in \mathbf{T}$ for all $x, y \in \Gamma$, that is, $v(x) \in \mathbf{T}^{f \sigma_{2}}$ for all $x \in \Gamma$, and thus $v \in Z_{f, \sigma_{2}}^{1}\left(\Gamma, \mathbf{T}^{f \sigma_{2}}\right)$. By definition, both equalities above hold if and only if $\sigma_{1} \sim \sigma_{2}$ via the maps $\tau \in \operatorname{Aut}(\Gamma)$, $f \in \operatorname{qAut}(G)$ and $v \in Z_{f, \sigma_{2}}^{1}\left(\Gamma, \mathbf{T}^{f \sigma_{2}}\right)$.

Observe that $\operatorname{Aut}(G)$ acts on $\operatorname{Emb}(\Gamma, G)$ by $f \circ \sigma$ for all $f \in \operatorname{Aut}(G)$ and $\sigma \in \operatorname{Emb}(\Gamma, G)$. In particular, $G$ and $\mathbf{T}$ act on $\operatorname{Emb}(\Gamma, G)$ by $\operatorname{Int}(G)$ and $\operatorname{Int}(\mathbf{T})$ respectively. Denote $\operatorname{Int}(g) \circ \sigma=g \cdot \sigma$, for all $\sigma \in \operatorname{Emb}(\Gamma, G), g \in G$ and $G \cdot \sigma$ the orbit of $\sigma$ in $\operatorname{Emb}(\Gamma, G)$ under the action of $G$. Clearly, if $C=C_{G}(\sigma(\Gamma))$ is the centralizer of $\sigma(\Gamma)$ in $G$, then $G \cdot \sigma \simeq G / C$; and the set of $\mathbf{T}$-orbits in $G \cdot \sigma$ is $\mathbf{T} \backslash G / C$.

Lemma 4.3.3. If $\sigma(\Gamma)$ is not central in $G$, then $\mathbf{T} \backslash G / C$ is infinite.

Proof. To prove that the set $\mathbf{T} \backslash G / C$ is infinite it is enough to show that $\operatorname{dim} G>\operatorname{dim} \mathbf{T}+\operatorname{dim} C=\operatorname{rk} G+\operatorname{dim} C$, since if $m=\# \mathbf{T} \backslash G / C$ were finite, then $G=\bigcup_{i=1}^{m} \mathbf{T} g_{i} C$ and this would imply that $\operatorname{dim} G \leq \operatorname{dim} \mathbf{T}+\operatorname{dim} C$. Since $\sigma(\Gamma)$ is not central in $G$, there exists $g \in \sigma(\Gamma)$ non-central; then the centralizer of $g$ is a proper reductive subgroup of $G$ by $[\mathrm{R}]$. Hence $C$ is contained in a maximal reductive subgroup $M$ of $G$. Let $\mathfrak{g}$ and $\mathfrak{m}$ be the Lie algebras of $G$ and $M$ respectively. As the maximal subalgebras of the simple Lie algebras were classified by Dynkin, by inspection in D1, D2 one can 
see that $\operatorname{dim} \mathfrak{g}>\operatorname{rk} \mathfrak{g}+\operatorname{dim} \mathfrak{m}$ for any maximal reductive $\mathfrak{m}$ and the lemma follows.

Lemma 4.3.4. If $\sigma(\Gamma)$ is not central in $G$, then there exists an infinite family $\left\{g_{i}\right\}_{i \in I}$ of elements of $G$ such that $\left(g_{i} \cdot \sigma\right)(\Gamma) \nsubseteq \mathbf{T}$.

Proof. Let $\left\{g_{j}\right\}_{j \in J}$ be a set of representatives of $\mathbf{T} \backslash G / C$. Then, $J$ is infinite by Lemma 4.3.3. We will prove that there can only be finitely many $g_{j}$ such that $\left(g_{j} \cdot \sigma\right)(\Gamma) \subseteq \mathbf{T}$.

Suppose that there exists $g_{i} \in G$ such that $\left(g_{i} \cdot \sigma\right)(\Gamma) \subseteq \mathbf{T}$. Without loss of generality we can assume that $\Gamma \subseteq \mathbf{T}$. Consider the sets

$$
\mathcal{L}=\left\{h \in G \mid h \Gamma h^{-1} \subseteq \mathbf{T}\right\} \quad \text { and } \quad \Lambda=\sum_{h \in \mathcal{L}} h \Gamma h^{-1} .
$$

Then clearly $\mathbf{T} \subseteq \mathcal{L}$ and $\Lambda \subset \mathbf{T}$. We show that $\mathbf{T} \backslash \mathcal{L} / C$ is finite. Let $N=|\Gamma|$, then $N \Lambda=0$, which implies that $\Lambda \subseteq\left(\mathbb{G}_{N}\right)^{n}$, where $\mathbb{G}_{N}$ is the group of $N$-th roots of unity. Thus $\Lambda$ is a finite subgroup of $\mathbf{T}$. In particular, it contains only finitely many subgroups which are pairwise distinct and isomorphic to $\Gamma$. Let $\Gamma_{1}, \ldots, \Gamma_{s}$ be these subgroups and $h_{i} \in \mathcal{L}$ such that $h_{i} \Gamma h_{i}^{-1}=\Gamma_{i}$. If $h \in \mathcal{L}$, then $h \Gamma h^{-1}=h_{i} \Gamma h_{i}^{-1}$ for some $1 \leq i \leq s$. Hence $h_{i}^{-1} h \Gamma h^{-1} h_{i}=\Gamma$ and $h_{i}^{-1} h \in N_{G}(\Gamma)$, the normalizer of $\Gamma$ in $G$. Thus $\mathcal{L}=\coprod_{i=1}^{s} h_{i} N_{G}(\Gamma)$.

On the other hand, there is a homomorphism $N_{G}(\Gamma) \rightarrow \operatorname{Aut}(\Gamma)$ which factorizes through the monomorphism $N_{G}(\Gamma) / C \hookrightarrow \operatorname{Aut}(\Gamma)$. Since $\Gamma$ is finite, the order of $N_{G}(\Gamma) / C$ is finite and consequently $\mathcal{L} / C$ is finite. Since by assumption $\Gamma \subseteq \mathbf{T}$, there exists an injection $\mathbf{T} \backslash \mathcal{L} / C \rightarrow \mathcal{L} / C$, which implies that $\left\{g_{j}\right\}_{j \in J}$ contains only finitely many elements such that $g_{j} \Gamma g_{j}^{-1} \subseteq \mathbf{T}$.

Now we are able to prove our main theorem.

Theorem 4.3.5. Let $\sigma \in \operatorname{Emb}(\Gamma, G)$ such that $\sigma(\Gamma)$ is not central in $G$. Then there exists an infinite family $\left\{\sigma_{j}\right\}_{j \in J} \subset \operatorname{Emb}(\Gamma, G)$ such that the Hopf algebras $\left\{A_{\sigma_{j}}\right\}_{j \in J}$ of dimension $|\Gamma| \ell^{\mathrm{dim} \mathfrak{g}}$ are pairwise non-isomorphic, non-semisimple, non-pointed and their duals are also non-pointed.

Proof. Since $\sigma(\Gamma)$ is not central in $G$, from Lemma 4.3.3 it follows that there exists an infinite set $\left\{g_{i}\right\}_{i \in I}$ of elements in $G$ such that $\left(t g_{i}\right) \cdot \sigma \neq g_{j} \cdot \sigma$ for all $i \neq j$ and $t \in \mathbf{T}$. Denote $\sigma_{i}=g_{i} \cdot \sigma$ for all $i \in I$. By Lemma 4.2.13 we know that

$$
\operatorname{Emb}(\Gamma, G)=\coprod_{\eta \in E} \mathfrak{C}_{\eta},
$$

where $\mathfrak{C}_{\eta}=\{\mu \in \operatorname{Emb}(\Gamma, G) \mid \eta \sim \mu\}$ and $E$ is a set of representatives of $\operatorname{Emb}(\Gamma, G)$ under the equivalence relation $\sim$. We prove now that there can not be infinite embeddings $\sigma_{i}$ included in only one $\mathfrak{C}_{\eta}$.

Suppose on the contrary, that there exists $\eta \in \operatorname{Emb}(\Gamma, G)$ such that $\mathfrak{C}_{\eta}$ contains infinitely many $\sigma_{i}$. By definition and Theorem 4.3.2, we know that

$$
\mathfrak{C}_{\eta}=\bigcup_{\tau \in \operatorname{Aut}(\Gamma), f \in \operatorname{qAut}(G)} \mathfrak{C}_{\eta, f, \tau},
$$


where $\mathfrak{C}_{\eta, f, \tau}=\left\{\mu \in \operatorname{Emb}(\Gamma, G) \mid \eta \sim \mu\right.$ via $(\tau, f, v)$, some $\left.v \in Z_{f, \eta}^{1}\left(\Gamma, \mathbf{T}^{f \eta}\right)\right\}$.

By Lemma 4.2.7. $\mathbf{T}$ and $\mathbf{T}^{f \eta}$ act on $q \operatorname{Aut}(G)$ by $t \cdot f(x)=t f(x) t^{-1}$ for all $f \in \operatorname{qAut}(G), t \in \mathbf{T}$. We claim that if $t \in \mathbf{T}^{f \eta}$, then $\mathfrak{C}_{\eta, t \cdot f, \tau}=\mathfrak{C}_{\eta, f, \tau}$.

Indeed, $\mu \in \mathfrak{C}_{\eta, t \cdot f, \tau}$ if and only if there exists $v \in Z_{(t \cdot f), \eta}^{1}\left(\Gamma, \mathbf{T}^{(t \cdot f) \eta}\right)$ such that $\mu(\tau(x))=(t \cdot f)(\eta(x)) v(x), x \in \Gamma$. But in such a case

$$
\begin{aligned}
\mu(\tau(x)) & =(t \cdot f)(\eta(x)) v(x)=t f(\eta(x)) t^{-1} v(x)=t f(\eta(x)) v(x) t^{-1} \\
& =f(\eta(x))\left[f(\eta(x))^{-1} t f(\eta(x))\right] v(x) t^{-1}=f(\eta(x))(t \cdot v)(x),
\end{aligned}
$$

which implies that $\mu \in \mathfrak{C}_{\eta, f, \tau}$, since by Lemma 4.2.15, $Z_{(t \cdot f), \eta}^{1}\left(\Gamma, \mathbf{T}^{(t \cdot f) \eta}\right)=$ $Z_{f, \eta}^{1}\left(\Gamma, \mathbf{T}^{f \eta}\right)$ for all $t \in \mathbf{T}$, and by Lemma 4.2.16 $t \cdot v \in Z_{f, \eta}^{1}\left(\Gamma, \mathbf{T}^{f \eta}\right)$. Thus, $\mathfrak{C}_{\eta, t \cdot f, \tau} \subseteq \mathfrak{C}_{\eta, f, \tau}$ and similarly for the other inclusion. Hence we can write

$$
\mathfrak{C}_{\eta}=\coprod_{\tau \in \operatorname{Aut}(\Gamma)} \coprod_{f \in \mathfrak{J}} \coprod_{t \in \mathfrak{t}} \mathfrak{C}_{\eta, t \cdot f, \tau}
$$

where $\mathfrak{J}$ is a set of representatives of qAut $(G) / \mathbf{T}$ and $\mathfrak{t}$ is a set of representatives of $\mathbf{T} / \mathbf{T}^{f \eta}$. Since $\operatorname{Aut}(\Gamma)$ is finite and by Lemma 4.2.7 (c), $\mathfrak{J}$ is also finite, if $\mathfrak{C}_{\eta}$ contains infinitely many $\sigma_{i}$ then there exist $\tau \in \operatorname{Aut}(\Gamma)$ and $f \in \mathfrak{J}$ such that $\coprod_{t \in \mathfrak{t}} \mathfrak{C}_{\eta, t \cdot f, \tau}$ contains infinite many $\sigma_{i}$. If $\sigma_{i} \in \mathfrak{C}_{\eta, t \cdot f, \tau}$ for some $t \in \mathfrak{t}$, then $t^{-1} \cdot \sigma_{i} \in \mathfrak{C}_{\eta, f, \tau}$. By Lemmas 4.2.16 and 4.2.18 we know that the set $\mathfrak{C}_{\eta, f, \tau} / \mathbf{T}^{f \eta}$ is finite, hence there must exists $\sigma_{j}, i \neq j$ and $s \in \mathbf{T}$ such that $\left[t^{-1} \cdot \sigma_{i}\right]=\left[s^{-1} \cdot \sigma_{j}\right]$. But this contradicts our assumption on the family $\left\{\sigma_{i}\right\}_{i \in I}$ since in such a case, there would exist $r \in \mathbf{T}^{f \eta}$ such that $t^{-1} \cdot \sigma_{i}=r \cdot\left(s^{-1} \cdot \sigma_{j}\right)=\left(r s^{-1}\right) \cdot \sigma_{j}$, that is $\sigma_{i}=\left(t r s^{-1}\right) \cdot \sigma_{j}$ with $t r s^{-1} \in \mathbf{T}$.

In conclusion, there can be only finite elements of the set $\left\{\sigma_{i}\right\}_{i \in I}$ in each $\mathfrak{C}_{\eta}$, for any $\eta \in E$. Thus by Theorems 4.1.7 and 4.3.2 and Lemma 4.3.4 there exists an infinite subset $J \subset I$ such that the Hopf algebras $\left\{A_{\sigma_{j}}\right\}_{j \in J}$ are pairwise non-isomorphic, non-semisimple, non-pointed, and with nonpointed duals.

Example 4.3.6. Let $n \geq 2$ and let $\Gamma$ be a finite subgroup of $S L(2)$. Let us consider $\sigma: \Gamma \rightarrow S L_{n}(\mathbb{C})$ given by $\sigma(x)=\left(\begin{array}{cc}x & 0 \\ 0 & I_{n-2}\end{array}\right), x \in \Gamma$. If $\Gamma$ is not central, that is $\Gamma \nsubseteq\{ \pm \mathrm{id}\}$, we get an infinite family of pairwise non-isomorphic Hopf algebras non-semisimple, non-pointed with non-pointed duals, of dimension $|\Gamma| \ell^{n^{2}-1}$. An analogous example can be described for any simple $G$.

Example 4.3.7. The examples given by Müller in [M3, Thm. 5.13] fits into the situation above for $\mathfrak{g}=\mathfrak{s l}_{2}$ and $\Gamma=\mathbb{G}_{\ell}$. He shows there by a different technique that the embedding given in the previous example gives an infinite family of pairwise non-isomorphic Hopf algebras as in Theorem 4.1.7.

Example 4.3.8. The finite subgroups of a simple affine algebraic group $G$ are not known. However, some positive results for subgroups of prime order were proved by several authors. 
Theorem 4.3.9. [S, Thm. 1] Let $G$ be a connected linear algebraic simple group over an algebraically closed field $k$. Let $h$ be its Coxeter number and let $p$ be a prime number.

(a) If $p=h+1$, the group $G(k)$ contains a subgroup isomorphic to $P G L_{2}\left(\mathbb{F}_{p}\right)$, with exception to char $k=2$ and to $G \simeq P G L_{2}$.

(b) If $p=2 h+1, G(k)$ contains a subgroup isomorphic to $P S L_{2}\left(\mathbb{F}_{p}\right)$.

The following results state when $G$ contains a cyclic subgroup of order $p$. By Theorem 4.1.7 we obtain Hopf algebras of dimension $p \cdot \ell^{\operatorname{dim} \mathfrak{g}}$.

Proposition 4.3.10. [S, Prop. 4] Assume that $G(k)$ is simple and let $h$ be the Coxeter number associated to $G$. If $p=m h+1$, for $m \geq 1$, then $G$ contains a regular element of order $p$.

Let $G$ be a compact connected simple Lie group. If $x \in G$ has order $N$, then the character values $\operatorname{tr}_{V}(\rho(x))$, as $(\rho, V)$ varies over all finite dimensional representations of $G$, generate a subfield $A(x)$ of $\mathbb{Q}(\xi)$, where $\xi=e^{2 \pi i / N}$. The degree of $\mathbb{Q}(\xi) / A(x)$ is called the depth of $x$.

Theorem 4.3.11. $[\mathrm{PW}$, Thm. 3] Let $p$ be a prime number not dividing $|W|$ and let $d$ be a positive integer. Then $G$ has an element of order $p$ and depth $d$ if and only if the following all hold:

(a) $d$ divides $p-1$.

(b) $d$ divides $m+1$ for some exponent $m$ of $W$.

(c) If $d$ is odd then $G$ is either of type $A_{l}$ or $E_{6}$ or $D_{l}$, with $l$ an odd multiple of $d$.

Acknowledgments. The authors thank S. Natale for helpful indications on Lemma 4.2.18 and J. Vargas for discussions on reductive subgroups of a simple group.

\section{REFERENCES}

[A] N. Andruskiewitsch, 'Notes on extensions of Hopf algebras', Can. J. Math. 48 (1996), no. 1, 3-42.

[AD] N. Andruskiewitsch and J. Devoto, 'Extensions of Hopf algebras', St. Petersburg Math. J. 7 (1996), no. 1, 17-52.

[AS1] N. Andruskiewitsch and H-J. Schneider, Appendix to A.

[AS2] 'On the classification of finite-dimensional pointed Hopf algebras'. Ann. Math., to appear.

[Br] K. S. Brown, Cohomology of Groups, Springer-Verlag (1982).

[BG] K. A. Brown and K. R. Goodearl, Lectures on Algebraic Quantum Groups, Advanced Courses in Mathematics - CRM Barcelona. Basel: Birkhäuser.

[DK] C. De Concini and V. G. KAC, 'Representations of quantum groups at roots of 1', Colloque en honeur a J. Dixmier, Paris (1990) Prog. Math. 92, 471-506.

[DKP] C. De Concini, V. G. KaC and C. Procesi 'Quantum coadjoint action', J. Am. Math. Soc. 5 (1992), no. 1, 151-189.

[DL] C. De Concini and V. Lyubashenko, 'Quantum function algebra at roots of 1', Adv. Math. 108 (1994), no. 2, 205-262.

[DT] Y. Doi and M. TAKeuchi, 'Cleft comodule algebras for a bialgebra', Commun. Algebra 14 (1986), 801-818. 
[D1] E. B. Dynkin, 'Semisimple subalgebras of semisimple Lie algebras', Am. Math. Soc., Transl., II. Ser. 6 (1957), 111-243.

[D2] 'Maximal subgroups of the classical groups', Am. Math. Soc., Transl., II. Ser. 6 (1957), 245-378.

[G1] G. A. GarcíA, 'On Hopf algebras of dimension $p^{3}$, Tsukuba J. Math. 29 (2005), no. 1, 259-284.

[G2] Tesis de doctorado, Universidad de Córdoba, available in March 2007.

$[\mathrm{H}]$ G. Hochschild, 'Algebraic groups and Hopf algebras', Ill. J. Math. 14 (1970), $52-65$.

[Hf] I. Hofstetter, 'Extensions of Hopf algebras and their cohomological description', J. Algebra 164 (1994), no. 1, 264-298.

[Hu] J.E. Humphreys, Introduction to Lie Algebras and Representation Theory, GTM 9 (1980). New York - Heidelberg - Berlin: Springer-Verlag. XII

[Hu2] L Linear Algebraic Groups, Graduate Texts in Mathematics, 21 (1981). New York - Heidelberg - Berlin: Springer-Verlag. XVI

[J] J.C. Jantzen, Lectures on Quantum Groups, Graduate Studies in Mathematics. 6 (1996). Providence, RI: American Mathematical Society (AMS).

[MS] S. MAJID and YA. S. SolBelman, 'Bicrossproduct structure of the quantum Weyl group', J. Algebra 163 (1994), no. 1, 68-87.

[Mk] A. Masuoka, 'Defending the negated Kaplansky conjecture', Proc. Am. Math. Soc. 129 (2001), no. 11, 3185-3192.

[Mo] S. Montgomery, Hopf Algebras and their Actions on Rings, CBMS Reg. Conf. Ser. Math. 82 (1993). American Mathematical Society (AMS).

[M1] E. MüLlER, 'Some topics on Frobenius-Lusztig kernels I', J. Algebra 206 (1998), 624-658.

[M2] , 'Some topics on Frobenius-Lusztig kernels II', J. Algebra 206 (1998), 659681.

[M3] 'Finite subgroups of the quantum general linear group', Proc. London Math. Soc. (3) 81 (2000), no. 1, 190-210.

[NZ] W.D. Nichols and M. B. Zoeller, 'Freeness of infinite dimensional Hopf algebras over grouplike subalgebras', Comm. Algebra 17 (1989), no. 2, 413-424.

[PW] A. Pianzola and A. Weiss, 'The Rationality of Elements of Prime Order in Compact Connected Simple Lie Groups', J. Algebra 144 (1991), 510-521.

[R] Richardson, R.W., 'Orbits, invariants, and representations associated to involutions of reductive groups' Invent. Math. 66, 287-312 (1982).

[S] J. P. SERre, 'Exemples de plongements des groups $P S L_{2}\left(F_{p}\right)$ dans des groupes de Lie simples', Invent. Math. 124 (1996), no. 1-3, 525-562.

[Sch1] H-J. SchneIDER, 'Some remarks on exact sequences of quantum groups', Commun. Algebra 21 (1993), no. 9, 3337-3357.

[Sch2] , 'Normal basis and transitivity of crossed products for Hopf algebras', $J$. Algebra 152 (1992), no. 2, 289-312.

[Sw] M. Sweedler, 'Hopf algebras', Benjamin, New York, 1969.

Famaf-Ciem, Universidad Nacional de Córdoba Medina Allende s/n, Ciudad Universitaria, 5000 Córdoba, República Argentina.

E-mail address: andrus@mate.uncor.edu, ggarcia@mate.uncor.edu 